\title{
Motion Law of Graviton in Three-Dimensional Space and Its Influence on Star Distribution of Spiral Galaxy
}

\author{
Pingan Luo \\ Third Block, Yunqu Yuan, Huilong Guan, Changping District, Beijing, China \\ Email: luopingan6362@163.com, luopingan6362@sohu.com
}

Received 13 September 2014; revised 11 October 2014; accepted 8 November 2014

Copyright (C) 2014 by author and Scientific Research Publishing Inc.

This work is licensed under the Creative Commons Attribution International License (CC BY). http://creativecommons.org/licenses/by/4.0/

\section{(c) (i) Open Access}

\begin{abstract}
According to some fundamental hypotheses compatible with microphysics theory in allusion to property of graviton shot by the spiral galaxy, we set up a motion trace equation of graviton in the three-dimensional space, concluding method for measuring the velocity of graviton. This theory reveals formation mechanism of symmetric rotating arm image of the spiral galaxy. The rotating arm image conforms to Archimedes spiral. Under the condition of using dynamic constraints, the image that astrolabe is in thin-disk structure is naturally obtained. And then, we also deduce the law that astrolabe thickness $h$ is inversely proportional to the square root of the distance $r_{x y}$ between astrolabe and galaxy center, according with actual crossrange galaxy observation image excellently. This article, by utilizing several typical galaxy observation images with completely different appearances and shapes, verifies the motion trace law of graviton in three-dimensional space. And the theoretical simulation data meet observation image data very well. As per the discovery in the simulation of three-dimensional theoretical model, changes of parameters in theoretical model will exert a great influence on the shape of galaxy. This theory owns exclusively important meaning, not only to astrophysics, but also to reconstruction and development of relativity theory and the whole basic physics theory, as well as breaking through the current dilemma in studying graviton property.
\end{abstract}

\section{Keywords}

Graviton, The Velocity of Graviton, Motion Trace, Spiral Galaxy, M51, Symmetric Double Spiral Arm, Thin Disk

\section{Introduction}

As reported on websites of Cornell University, USA [1] and Nature, a British magazine [2] on Sept. 22, 2011, 
European researchers have discovered the superluminal velocity of neutrino, a phenomenon unexplainable. T. Adam [1] reveals that

1) Within a distance of 730 kilometers, the neutrinos are detected to travel 60 ns faster than light; In another word, neutrinos travel more 6 kilometers than light every second.

2) There is a relationship between travelling velocity and energy of neutrinos.

In June 2012, scientists from European Organization for Nuclear Research (CERN) made clarification that, the previous experimental results were wrong, and the problem happening to cable port of experimental facility led to occurrence of error [3].

As per Einstein's special theory of relativity, the speed of light is the limit of universal speeds, and any other speed cannot surpass light. Indeed, due to limitation of present experiment conditions, particle travelling faster than light has not been measured so far, but, it does not mean that there is no such particle, or guarantee that the particle with such property will not be discovered in the future. So, if "XX particle travels faster than light" is verified to be true, the classic theory of Einstein's will be revised. If the phenomenon of "XX particle travels faster than light" exists, does the velocity of graviton travel faster than light? How to measure? Is there any experimental observation datum to support?

Answers to the above questions, also come down to difficulties haunting modern physics for a long time, i.e., where is graviton? Which property it has? What is its velocity? Why cannot discover it in experiment? No matter whether graviton velocity surpasses light or not, physicists all know that the property of graviton may greatly affect construction of the whole physical theory. In today that graviton still cannot be measured, it is not proper to simply think that, the velocity of graviton is just light or must not exceed light.

Because universal gravitation is extremely weak, it cannot be found in the present experimental condition. In my article [4] published in 2012, I turned to astronomy from graviton research in viewing angle, firstly deriving motion law of graviton of the spiral galaxy in two-dimensional space, and proposing method of utilizing galaxy observation data to measure the velocity of graviton. The document [4], also points out, a correction method of Lorentz transformation in case of superluminal particle, which is the simplest and causes the fewest change to the present theory. Of course, it needs support of experimental data. In my article [5] published in 2013, I conduct experimental verification on motion law of graviton in the spiral galaxy raised in the document [4] and method for measuring graviton velocity, by using observation data [6] [7] of M51 galaxy, with the result showing that the theory and experiment accord with each other greatly.

In documents [4] [5], the motion law of graviton in the spiral galaxy and method for measuring graviton velocity stated are shown as follows

Supposed:

1) In any inertial reference system, the velocity of graviton in different directions shot is identical-theory of constant graviton velocity, that is, graviton owns property similar as photon.

2) The equivalent center of some spiral galaxy rotates with a constant rotational angular velocity $\omega$, with unit as radian/s. Attention: due to difference in direction of rotation axis, a plus-minus sign may exist.

3) Graviton shot outward by the spiral galaxy, mainly comes from the equivalent center of galaxy, as the major part in forming the gravitational field of the galaxy. Other stars are in such gravitational field.

4) The shooting processes of different gravitons are mutually independently.

5) In motion process, different gravitons may exert interaction effect each other, which can be omitted.

6) In the galaxy, peripheral stars are in normal distribution along the perpendicular line of motion trace of graviton.

So, the motion of graviton should meet the formula (1), getting the velocity of graviton via the formula (2).

$$
\begin{aligned}
& r=v_{\text {graviton }} \frac{\theta-\theta_{0}}{\omega} \\
& v_{\text {graviton }}=\frac{r \omega}{\theta-\theta_{0}}
\end{aligned}
$$

wherein, $v_{\text {graviton }}$ : Graviton velocity (m/s), or (light year/s).

$\theta$ : Rotating angle of equivalent center of galaxy (radian).

$\theta_{0}$ : Start rotating angle of equivalent center of galaxy (radian).

$r$ : Distance (m) between graviton and galaxy center, or (light year). 
$\omega$ : The velocity of rotating angle (radian/s) of equivalent center playing a leading role in spiral galaxy, may be faster than that of galactic center surface in spiral galaxy observed optically, but able to confirm lower limit of graviton velocity in accordance with the velocity of rotating angle of galactic center surface.

The characteristics of this theory (please see hypothesis 1 and 2), accord with "Archimedes Spiral" [8] in mathematics. Archimedes Spiral described in the document [8] writes, " $r=a \theta$. A motion point travels at constant speed $v$ along a radial, while the radial rotates toward pole o with constant angular velocity $\omega$. This curve is a trace formed by the motion point. The proportionality coefficient is got by $a=\frac{v}{\omega}$ ”.

With application of this theory into M51 spiral galaxy, observing data via M51 galaxy, several important formulas are obtained, which can be seen in formula (3') and (4'). As to detailed data processing procedure, please see the primary source [5]. Knowing from data analysis, the curve of rotating arm 1 described in this theory meets actual image perfectly, as shown in Figure 1.

$$
r=4.90 \times 10^{3} \theta+1.06 \times 10^{4}
$$

Unit: $r$ : light year, $\theta$ : radian.

$$
\frac{v_{\text {graviton }}}{\omega}=1.52 \times 10^{11}
$$

Unit: $v_{\text {graviton }}$ : Graviton velocity, unit: c, denoting light speed.

$\omega$ : The equivalent center's angular rotation velocity with a leading role in the spiral galaxy, may be faster than angular rotation velocity of galactic center surface in the spiral galaxy observed optically. Unit: radian/s.

By reason of discovering shell structure in M51 rotating arm afterwards, we deduct data of mutation area for transformation between shell structures, solve the formula (3') and (4'), and then get the formula (3) and (4). Later on, when processing data, the formula (3) and (4) will be taken as the criterion. In order to saving writing length, this part is omitted.

$$
\begin{gathered}
r=5.3812 \times 10^{3} \theta+1.0461 \times 10^{4} \\
\frac{v_{\text {graviton }}}{\omega}=1.6986 \times 10^{11}
\end{gathered}
$$

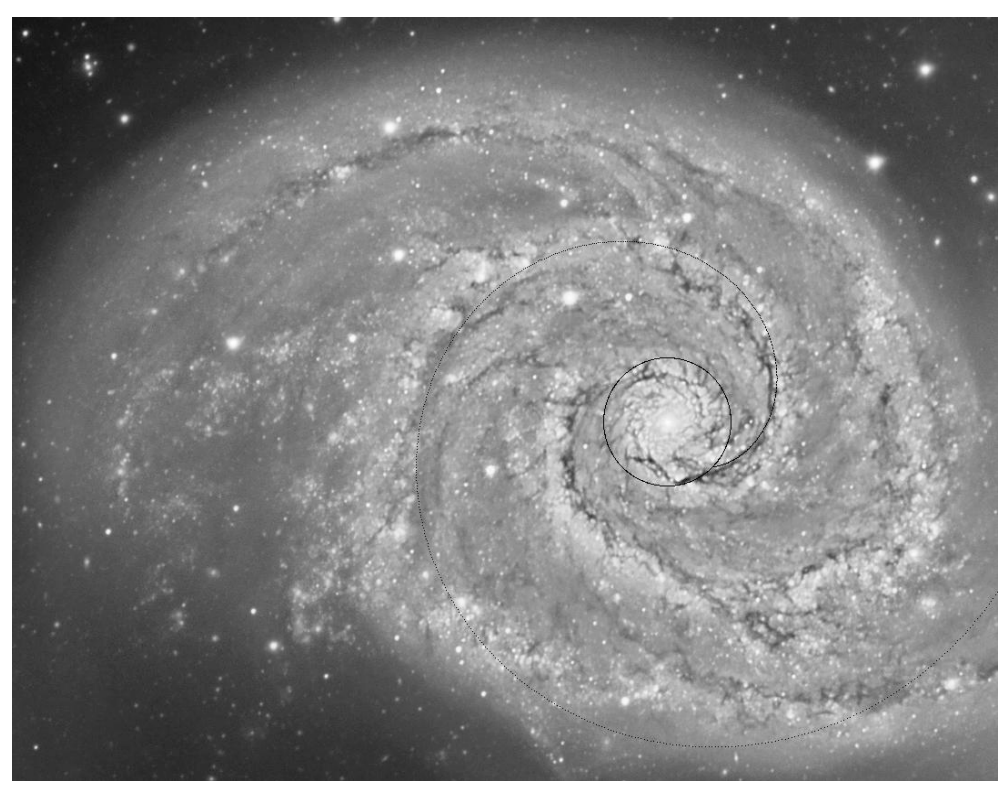

Figure 1. Comparison between motion trace of graviton described in fitting formula (3') and original picture. The curve is fitting curve, and the circle is boundary of galactic center marked by $r=70$ (pixel). 
Please note that, in the formula (3) and (4), implication and unit of every parameter and variable are same with that in the formula (3') and (4').

In short, via experimental observation data analysis, the document [5] gets the following particularly important conclusions,

1) The motion trace of graviton in the spiral galaxy accords with Archimedes spiral, perfectly meet observation data.

2) The guess that "the velocity of graviton is a constant" obtains support of observation data for the first time.

3) The upper limit of graviton velocity is obtained from observation data for the first time, i.e., the maximum velocity of graviton does not surpass 443,000 times of light speed.

4) Meanwhile, this article raises a possible approach to measure the lower limit of graviton velocity, and a new thought on utilizing observation data such as thickness of galactic center of the spiral galaxy to measure angular rotation velocity of the equivalent center.

5) The conclusion of this article gives out a significant prompt, reminding scientists studying graviton of not suffering from limitation of "the light speed is extremity" stated in the relativity theory, or ignoring analysis in various measurement signals before optical signal (optical signal is usually regarded as reference signal). Perhaps many significant discoveries occur in that time zone.

Although the conclusion that "the upper limit of graviton velocity does not surpass $4.43 \times 10^{5}$ times of the light speed" [5] is greatly astonishing, it is the first experimental data analysis report on graviton observation by human. The conclusions are all reached in accordance with actual observation data, and equipped with objectivity. Howsoever, as to human, from knowing little about the property of graviton, then conjecturing equivalence between the velocity of graviton and that of light, and finally gaining its quantitative analysis data, it is a great advance with extremely large significance.

In spite of the document [5] meets with great success, it belongs to the biaxial theory, losing lots of information concerning three-dimensional space. This article aims at, studying motion law of graviton in the three-dimensional spiral galaxy, revealing formation and distribution law of the spiral galaxy and verifying them through experimental observation image. From the following research, numerous characteristics of mysterious spiral galaxy can be found. These followed laws, actually, are very simple and nature.

The Section 2 below is to establish the physical model for graviton movement of spiral galaxy in the threedimensional space, explore the influence of change of several key parameters in the model on the disc shape of spiral galaxy and use the observation image to explain the corresponding galaxy pattern also exists in reality. Section 2.1, Establishing three-dimensional physical model; Section 2.2, taking the observation image of M51 galaxy as an example to obtain the parameter of the corresponding physical model; Section 2.3, discussing the influence of the parameter change of physical model on the disc shape of galaxy, for example, differential change of rotational angular velocity “ $\omega$ ” can generate a typical spiral galaxy, or barred spiral galaxy or ring galaxy; when other parameters remain the same, different initial value of rotation angle " $\theta$ " can cause the galaxy to be spiral or ring; Section 2.4, discussing the influence of value range of field angle " $\varphi$ " of three-dimensional reference system on the number of arm of spiral galaxy, namely, the essence for spiral galaxy to present doublearm pattern is the natural consequence for which the graviton rotates and emit into $4 \pi$ space at the same time.

The Section 3 is to research that force equilibrium condition is the intrinsic internal factor for spiral galaxy to form a think disc shape. At first, establishing the physical model of force equilibrium conditions of dynamics; Section 3.1, Based on the stable equilibrium conditions parallel to the direction of astrolabe plane to deduce general conditions that stars in the galaxy shall meet. When $\varphi=90^{\circ}$, such general condition is a famous kepler's third law; Section 3.2, researching the influence of stable equilibrium conditions parallel to the direction of astrolabe plane on the thickness of astrolabe and deducing the relationship between the thickness of astrolabe " $h$ " and the distance from the galaxy center " $r_{x y}$ ”; Section 3.3, Using the galaxy observation image to verify and comparing with the data of current mainstream theoretical model; finally, pointing out the existing law.

The Section 4 is to summarize the relevant conclusions obtained above.

\section{Motion Law of Graviton in the Spiral Galaxy and Natural Formation and Change of Symmetric Rotating Arm of the Spiral Galaxy in Three-Dimensional Space}

\subsection{Motion Trace of Graviton of the Spiral Galaxy in Three-Dimensional Space}

The actual spiral galaxy always has a certain thickness. In the previous research, the motion mechanism of gra- 
viton in the astrolabe axis direction has not been taken into account. So, in the next text, consider motion process of graviton in the astrolabe axis direction.

Supposed:

1) In any inertial reference system, the velocity of graviton in different directions shot is identical—-theory of constant graviton velocity, that is, graviton owns property similar as photon.

2) The equivalent center of some spiral galaxy rotates with a constant rotational angular velocity $\omega$, with unit as radian/s. Attention: due to difference in direction of rotation axis, a plus-minus sign may exist.

3) Graviton shot outward by the spiral galaxy, mainly comes from the equivalent center of galaxy, as the major part in forming the gravitational field of the galaxy. Other stars are in such gravitational field.

4) The shooting processes of different gravitons are mutually independently.

5) In motion process, different gravitons may exert interaction effect each other, which can be omitted.

6) In the galaxy, peripheral stars are in normal distribution along the perpendicular line of motion trace of graviton.

7) The motion of graviton shot in line with a certain shooting angle can be resolved into two motions. One is moving along principle plane paralleled to astrolabe (perpendicular to rotation axis of galaxy), and the other is moving toward the direction of rotation axis paralleled to galaxy.

8) Stabilizing celestial body nearby the motion curve of graviton is also in need of satisfying condition for force equilibrium in dynamics.

As shown in Figure 2, set up coordinate system. The equivalent center of spiral galaxy is in the original point $\mathrm{O}$ of coordinate system, and rotates around $Z$ axis at angular velocity $\omega$, and shoots graviton toward $\boldsymbol{r}$ (elevation angle $\varphi$, azimuth angle $\theta$ ). Its velocity is $V_{\text {graviton }} \cdot|\boldsymbol{r}|=V_{\text {graviton }} \cdot \Delta t, r_{x y}$ is projection of $\boldsymbol{r}$ on XOY plane. As per the above assumption, $\Delta t=\frac{\theta-\theta_{0}}{\omega}$, from parallelogram law of vector decomposition and resultant, we get,

$$
\left\{\begin{array}{l}
r_{x y}=\left(V_{\text {graviton }} \sin \varphi\right) \frac{\theta-\theta_{0}}{\omega} \\
h=\left(V_{\text {graviton }} \cos \varphi\right) \frac{\theta-\theta_{0}}{\omega} \\
\operatorname{tg} \varphi=\frac{r_{x y}}{h}
\end{array}\right.
$$

wherein,

$\varphi \in[0,2 \pi]$ : Elevation angle of shooting graviton, unit: rad (radian).

$\theta \in[0,+\infty)$ : Azimuth angle of shooting graviton, $\theta_{0}$ is initial-phase azimuth angle, able to be set as 0 , unit: rad (radian).

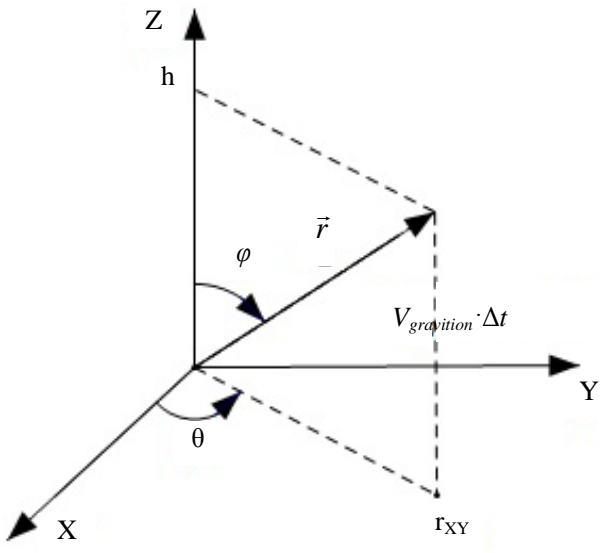

Figure 2. The equivalent center $\mathrm{O}$ of spiral galaxy shoots graviton in three-dimensional space. 
$r \in[0,+\infty)$ : Distance which graviton travel, unit: cs, using cs to express light second.

$z \in[0,+\infty)$ : Projection of distance which graviton travels in rotation axis, unit: cs, using cs to express light second.

$V_{\text {graviton }}$ : Motion rate of graviton, unit: c, using c to express light speed.

$\omega:$ The angular rotation velocity with leading role in the spiral galaxy may be faster than angular rotation velocity of galactic surface in spiral galaxy, which is a constant. Unit: rad/s (radian/second).

In order to simplify discussion process, just discuss $\theta \in[0,2 \pi]$.

\subsection{Quantization of Model Parameter}

According to in-depth and careful analysis in M51 galaxy data recently, a shell structure exists inside, so we make some modifications in allusion to the formula (3) and (4) stated in the document [5] (please refer to other special documents for details), just giving out results as shown in the formula (8) and (9) here.

$$
\begin{gathered}
r=5.3812 \times 10^{3} \theta+1.0461 \times 10^{4} \\
\frac{v_{\text {graviton }}}{\omega}=1.6986 \times 10^{11}
\end{gathered}
$$

This article applies revised formula, putting the formula (9) into the formula (5) and (6), and then obtaining that similar to the three-dimensional motion equation of graviton in M51 galaxy as follows,

$$
\left\{\begin{array}{l}
r_{x y}=\frac{1.6986 \times 10^{11}}{365 \times 24 \times 3600} \sin \varphi \cdot\left(\theta-\theta_{0}\right) \\
h=\frac{1.6986 \times 10^{11}}{365 \times 24 \times 3600} \cos \varphi \cdot\left(\theta-\theta_{0}\right) \\
\operatorname{tg} \varphi=\frac{r_{x y}}{h}
\end{array}\right.
$$

Here, units of $r_{x y}$ and $h$ are both light year, units of $\theta$ and $\varphi$ are both radian. Compare the formula (8) with (10), then get,

$$
\theta_{0}=\frac{-1.0461 \times 10^{4}}{1.6986 \times 10^{11} /(365 \times 24 \times 3600)}=-1.9422
$$

Please note: As for the formula (12), there should be one more constraint condition. Otherwise, in special condition, it will come up with $\frac{0}{0}$. So, we temporarily add a condition factitiously, i.e., $r_{x y}>r_{\min }$. Here, $r_{\min }$ is a boundary, smaller than it. Perhaps there is another element beginning to exert significant effect.

\subsection{Motion Trace and Variations of Graviton of the Spiral Galaxy in Three-Dimensional Space}

With the formula (10), (11) and (12), make use of Matlab to program and calculate it. The three-dimensional curve formed in line with motion trace of graviton is as shown in Figure 3 and the contour line is as shown in Figure 4.

From Figure 3, we can see 3D motion trace of graviton, i.e., possible location of celestial body. However, the ultimate location and distribution image of celestial body also suffer from constraint of kinetic equilibrium condition.

From Figure 4, we can see motion orbit of graviton in different heights ( $Z$ axis). In the plane where $Z=0$ is located, the contour lines is trace shown in 2D plane. Special attention: The middle of $Z=0$ contour line is disconnected, indicating that the galaxy center is not a barred galaxy and it accords with M51 galaxy very well (please see Figure 1).

1) The influence of change of angular rotation velocity $\omega$ on three-dimensional surface of graviton motion When $\omega$ decreases, $\frac{V_{\text {graviton }}}{\omega}$ will largen. Supposed that values in value comparison Figure 3 and Figure 4 


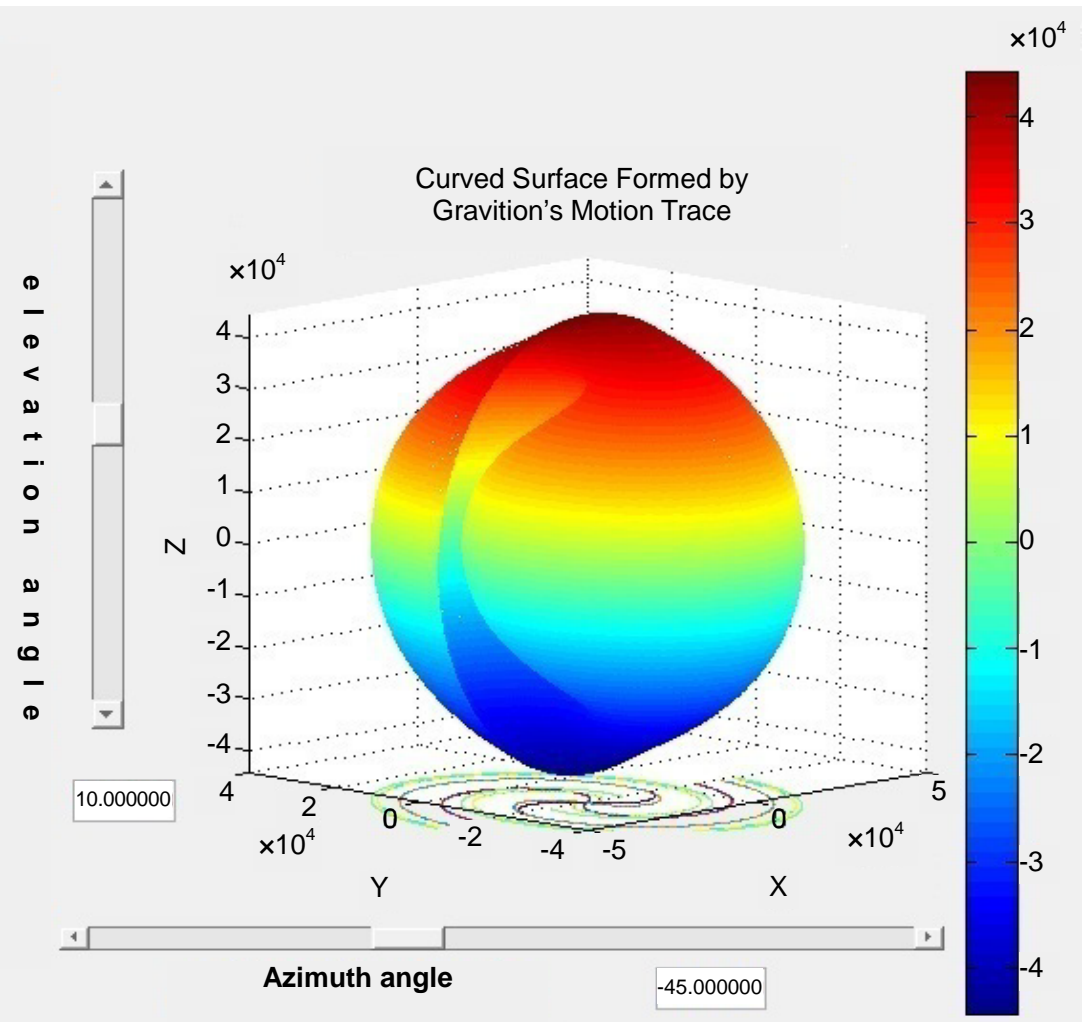

Figure 3. Curved surface formed by motion trace of graviton of spiral galaxy in three-dimensional space. Unit: light year.

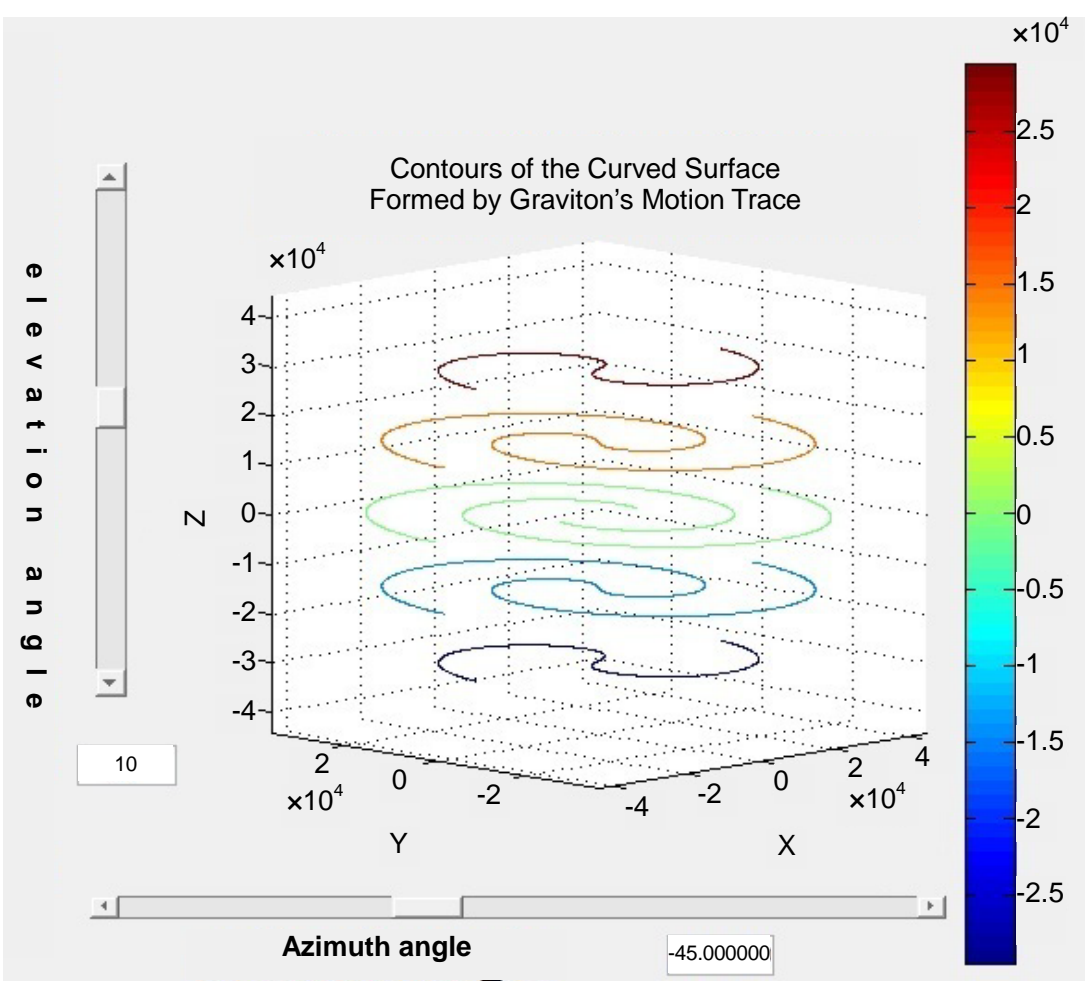

Figure 4. Contour lines of the curved surface formed by motion trace of graviton of spiral galaxy in three-dimensional space. Unit: light year. 
Of $\frac{V_{\text {graviton }}}{\omega}$ increase by 100 times, so the three-dimensional surface of graviton motion is as shown in Figure 5 and the contour lines are as shown in Figure 6. Please attentively see change of proportionality coefficient of scale data in coordinate axis.

From the contour line in $Z=0$ in Figure 6, the contour lines of its center has been connected in succession. It is supposed to be a typical example similar to barred spiral galaxy. Moreover, the distance unit of the contour lines with the same height increases by 100 times, which also means that the space between contour lines with the same height increases by 100 times.

When $\omega$ increases, $\frac{V_{\text {graviton }}}{\omega}$ will diminish. Supposed the values in value comparison Figure 3 and Figure 4 of $\frac{V_{\text {graviton }}}{\omega}$ decreases by 100 times, so the three-dimensional surface of graviton motion is as shown in Figure 7 and the contour lines are as shown in Figure 8.

Known from the contour lines in Figure 8, the shaped of contour lines in different heights have changed into annular appearance. If the angular rotation velocity of galactic center interior is comparatively large, when exceeding a certain numerical value, its contour line will be annular. Seen from the external, the galactic center just like being encircled by an oval light. That may be the formation mechanism of center shapes of may spiral galaxies.

The example concerning a actual galaxy: The living example that contour lines of galactic center shown in Figure 6, is just huge and beautiful barred spiral galaxy NGC1300. Silently lying in the side of Eridanus, it is far away from us for about 70 million light years [9]. Figure 9 shows image of gorgeous island universe obtained by compounding multiband shoot of Hubble, which is one of the largest complete galaxy image made throughout

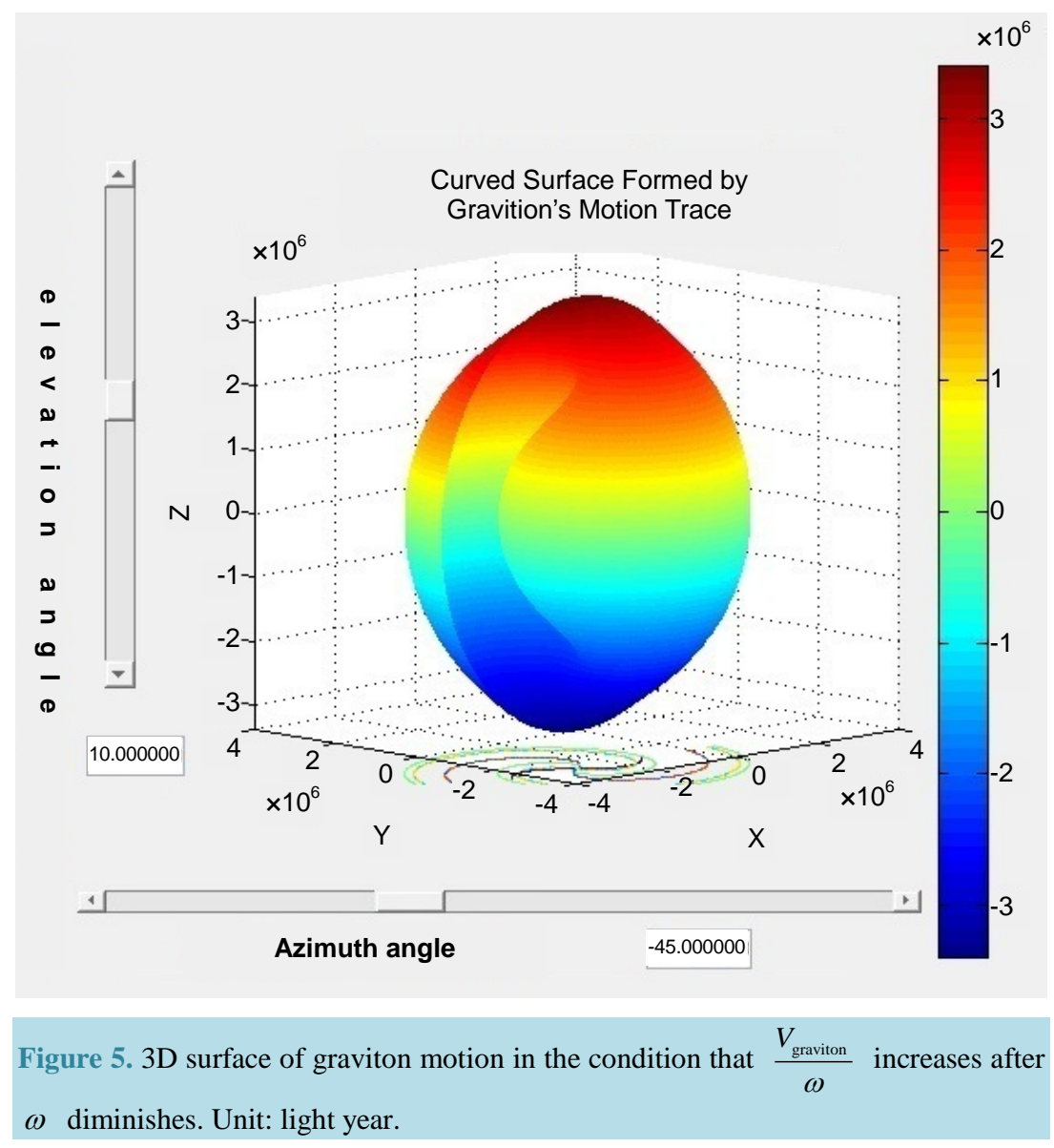




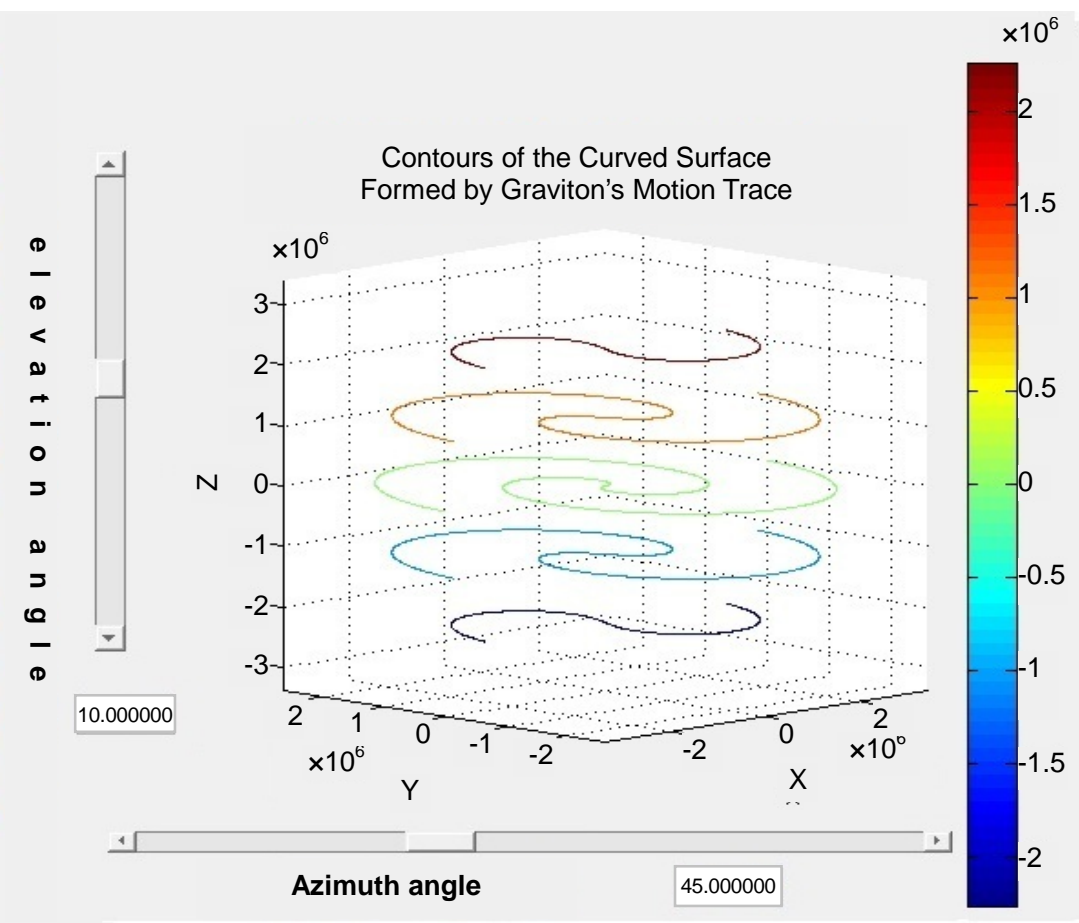

Figure 6. Contour lines in 3D surface of graviton motion in the condition that $\frac{V_{\text {graviton }}}{\omega}$ increases after $\omega$ diminishes. Unit: light year.

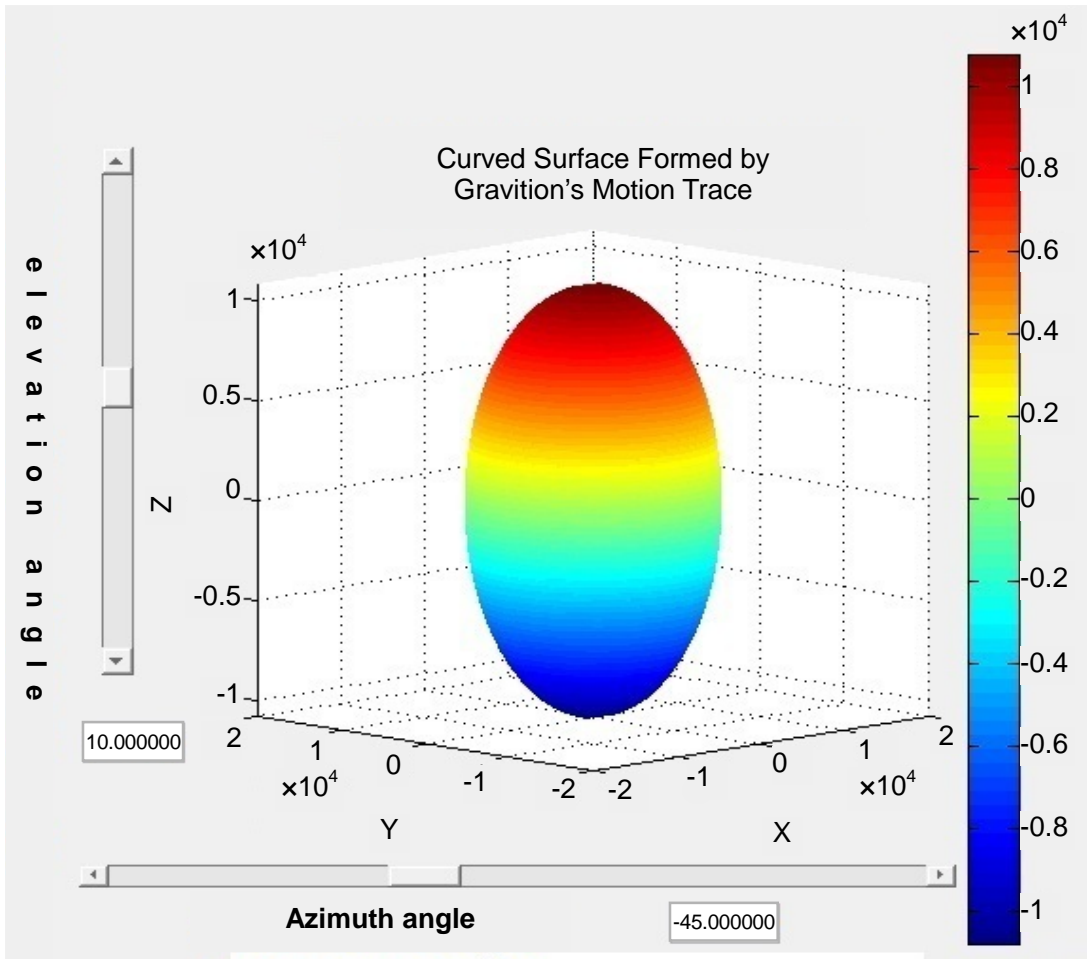

Figure 7. 3D surface in the condition that $\frac{V_{\text {graviton }}}{\omega}$ diminishes after $\omega$ increases. Unit: light year. 


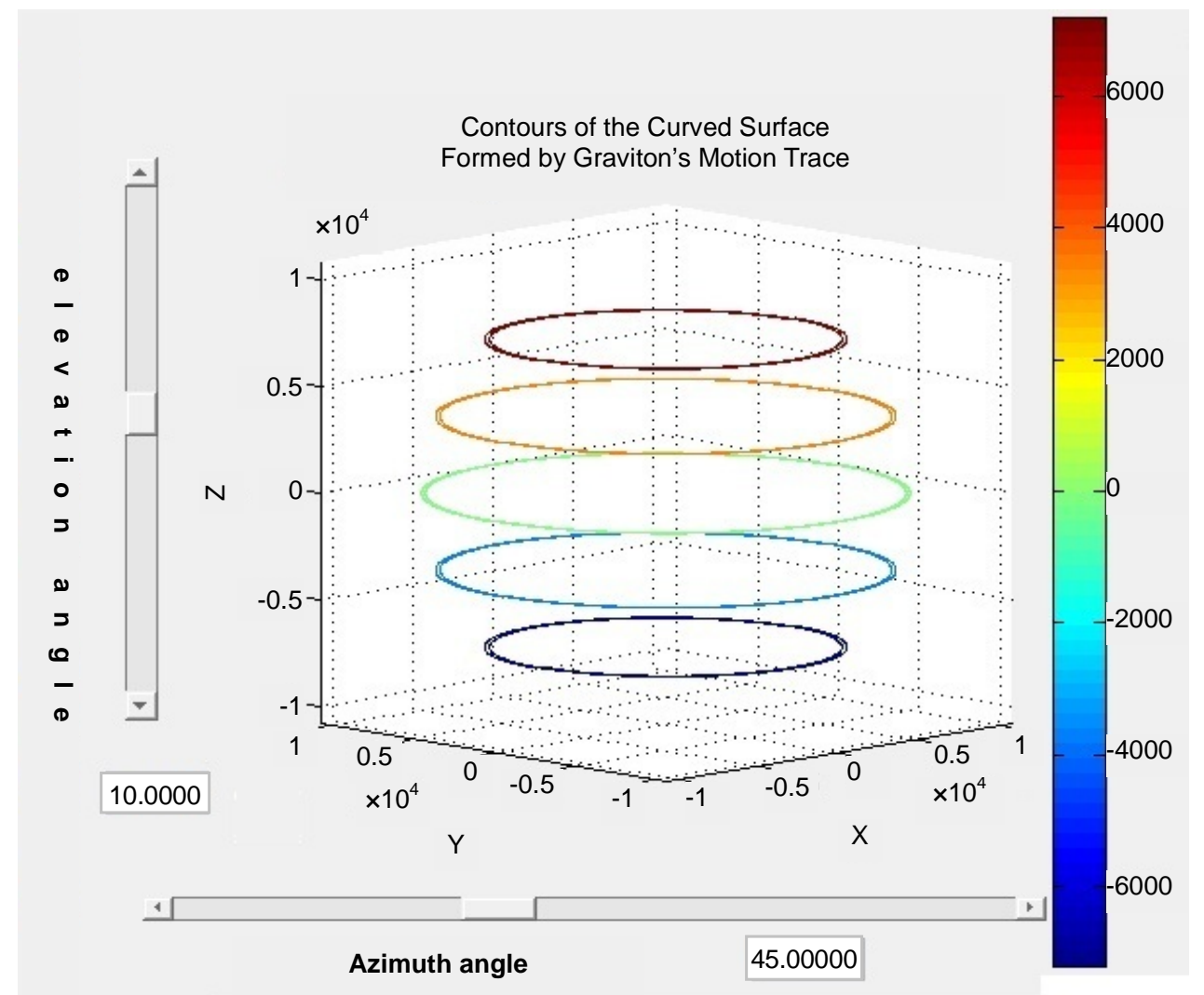

Figure 8. Contour lines in 3D surface of graviton motion in the condition that $\frac{V_{\text {graviton }}}{\omega}$ diminishes after $\omega$ increases. Unit: light year.

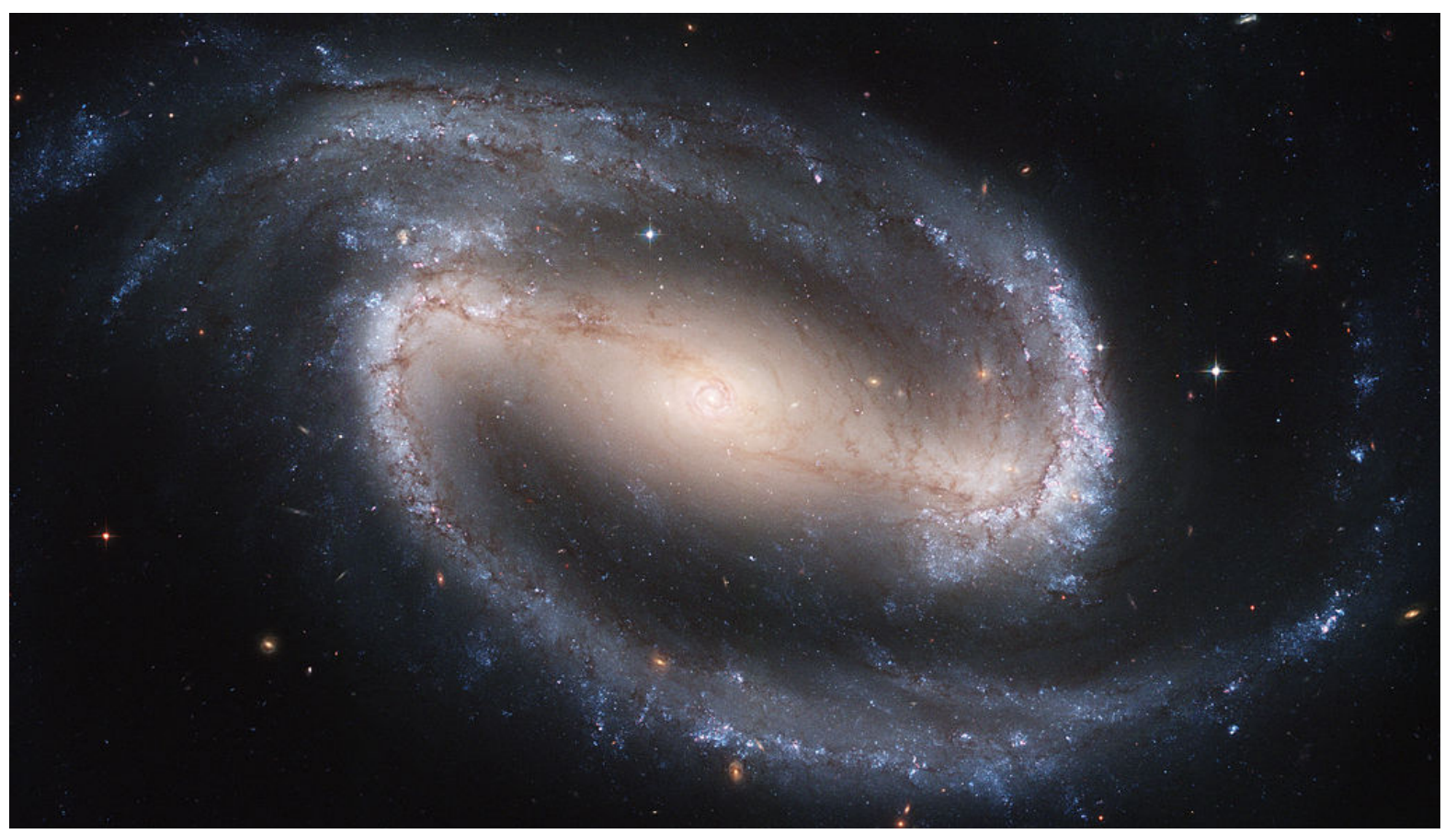

Figure 9. Picture of NGC1300 rodlike spiral galaxy. 
history (the original size of image: $6637 \times 3787$ ). NGC1300 stretches across 100,000 light years. This image shot by Hubble will play a leading role in indicating rodlike structure of the whole galaxy and perfectly display splendid spiral arms. Actually, if carefully check the hard core of this typical rodlike galaxy, you can find a obvious spiral structure area with span reaching 3000 light years.

There is also a living example in Figure 8. Please refer to Figure 12.

2) The influence of change of initial value of rotation angle $\theta$ on the three-dimensional surface of graviton motion

Except that the increase of rotation velocity will product ringlike contour line (as shown in Figure 8), via simulating calculation, we also find the influence of initial phase angle on formation of ringlike contour line. Addopt measurement data of M51 galaxy, i.e., making some changes in allusion to start angle in Figure 10 and Figure 11, such as letting $\theta_{0}=30 \mathrm{deg}$ so the three-dimensional surface of graviton motion is shown in Figure 10 and the contour line is shown in Figure 11. Attention: before modifying start angle, the image of contour lines are as shown in Figure 4, that is, contour lines are symmetric double spiral arms, and, in the plane that $Z=0$, the center of contour line is disconnected.

Seen from the contour lines in the position of $Z=0$ in Figure 11, the distribution of celestial bodies in galaxy approximately forms a circular ring.

As for Figure 8 and Figure 11, there is a living example too, that is, Art Hoag, an astronomer, accidentally found a extraordinary extragalactic system star-Hoag's Object [10], in 1950. Hoag's Object is an extremely well-known ring galaxy (as shown in Figure 12). Stretching across about 0.1 million light years, Hoag's Object is located in Serpens galaxy, far away from the earth for around 0.6 billion light years. Astrophiles and astronomers are interested in special appearance and distinctive structure of this galaxy, but as to the reason for formation of such galaxy, opinions vary, so there is still not a reasonable explanation to this day. According to motion trace theory of graviton, from Figure 8 and Figure 11, the angular rotation velocity is relatively faster, or start angle is comparatively bigger, which is its real internal cause.

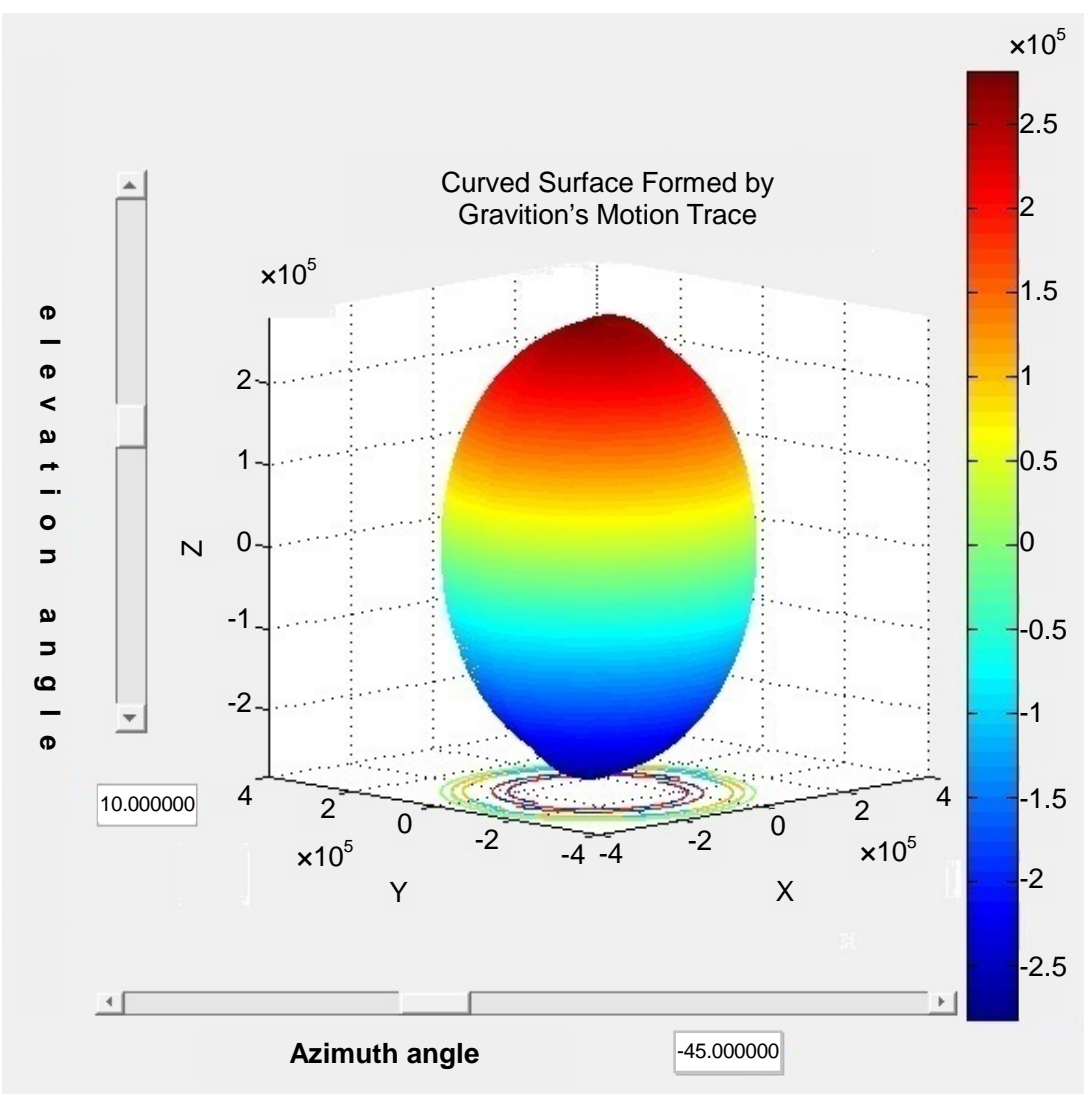

Figure 10. 3D surface of gravition motion when $\theta_{0}=30$ deg. Unit: light year. 


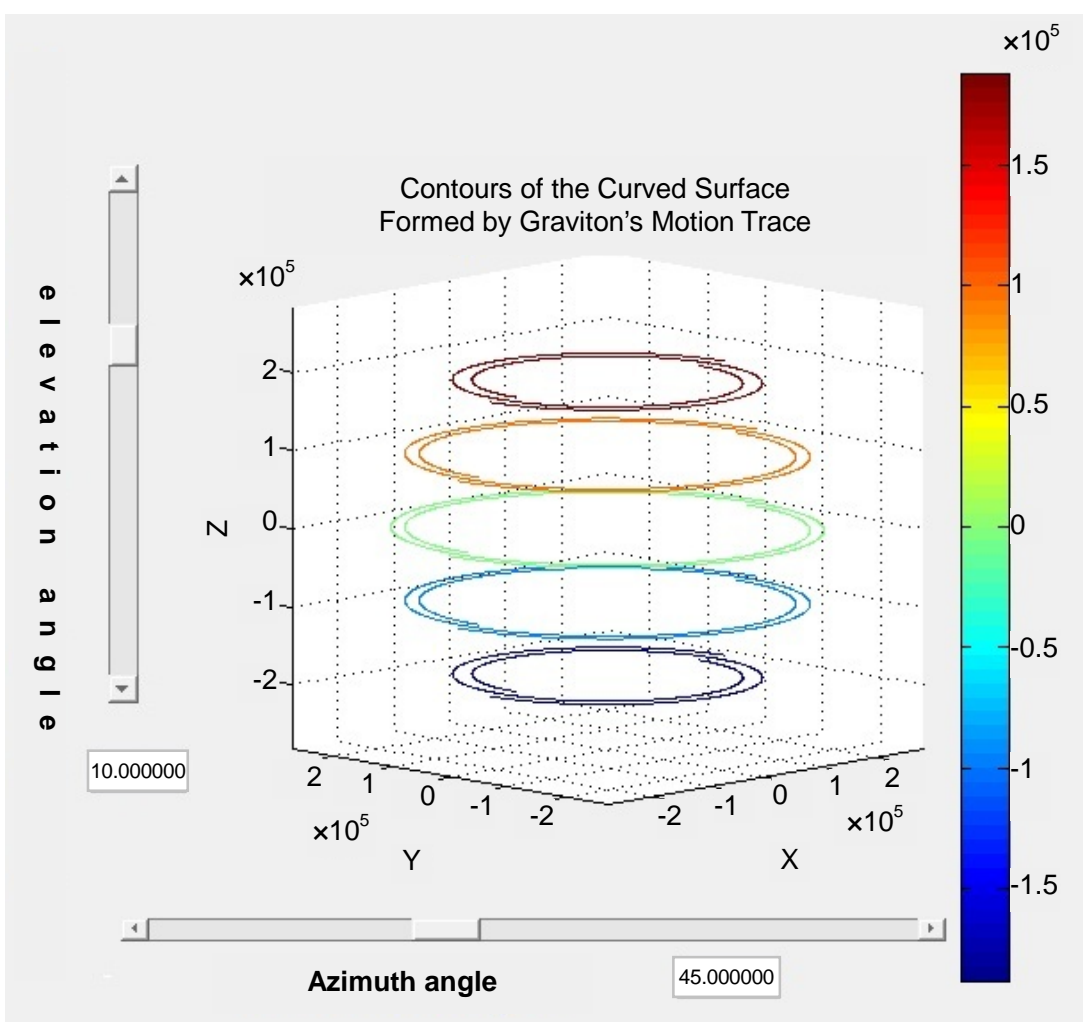

Figure 11. When $\theta_{0}=30 \mathrm{deg}$, the contour lines in 3D surface of graviton motion. Unit: light year.

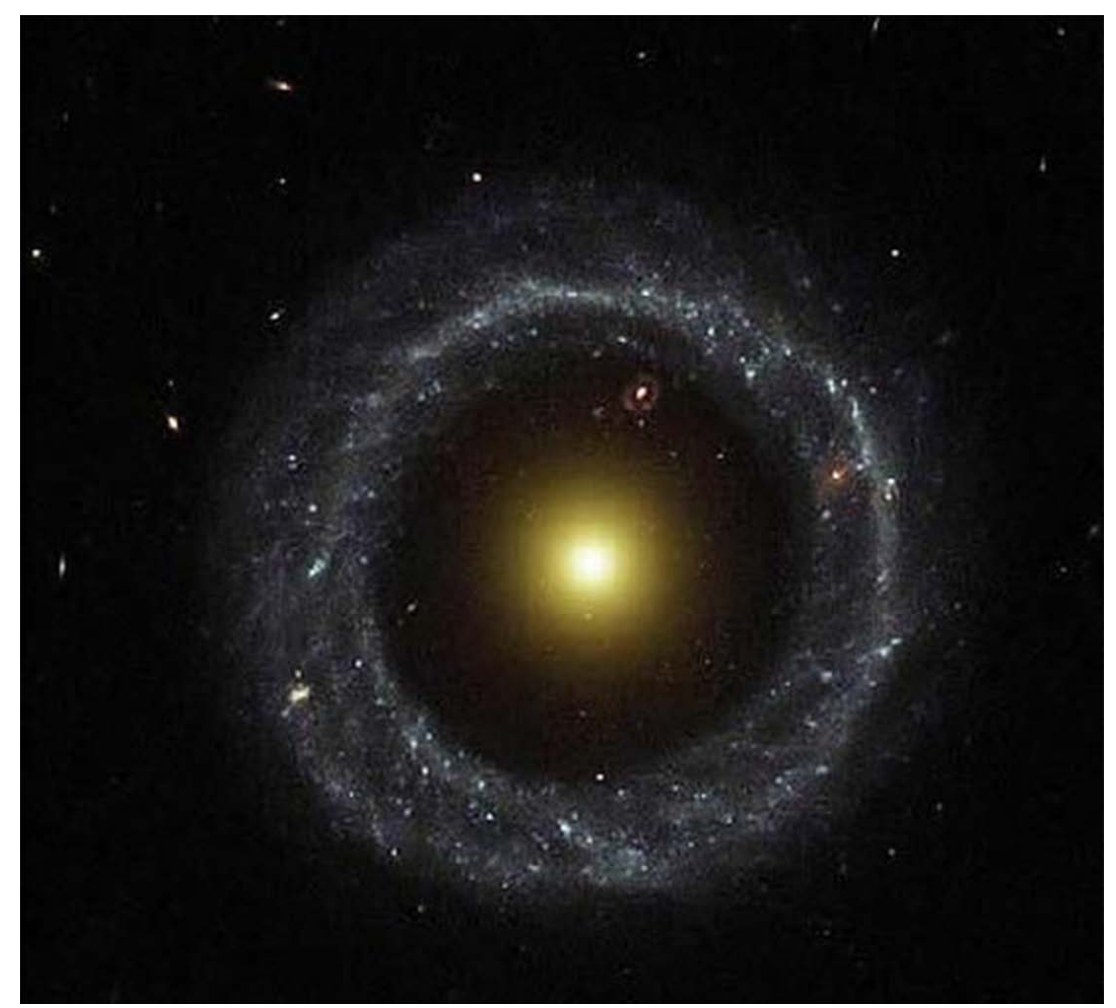

Figure 12. A extremely famous ring galaxy-Hoag's Object. 


\subsection{Mechanism Generated by Symmetric Double Rotating Arm Pattern of Spiral Galaxy}

The document [11] points out, "The first thing concerned in the astronomy community is that, why regular spiral pattern with large scale can exist and be kept in strong worse rotation system (such as strong worse rotation found in all non-barred spiral galaxy)? why such patterns mostly are symmetrical double arms?”

As per this theory, the regular spiral pattern generates naturally because of graviton motion trace, complying with the principle of Archimedes spiral. The symmetric double rotating arm pattern also comes into being naturally, and the reason for it is that graviton is shot toward $4 \pi$ space simultaneously at any time. Attention: In the previous analog computation, in the coordinate system in Figure 2, the value range of $\varphi$ is $\varphi \in[0,2 \pi]$, the pattern of symmetric double rotating arm will come up. If in line with the usual habit in setting up coordinate system, letting the value range of $\varphi$ be $\varphi \in[0, \pi]$ or $\varphi \in\left[-\frac{\pi}{2}, \frac{\pi}{2}\right]$, the pattern of single rotating arm will come up.

When $\varphi \in[0, \pi]$, the three-dimensional surface formed by graviton motion trace generated with the same algorithm and procedure is as shown in Figure 13, and the contour lines are as shown in Figure 14. Attention: a curve with the same altitude shown in Figure 14 is a spiral line, that is to say, Figure 13 and Figure 14 give out a single rotating arm pattern. Please compare Figure 3 with Figure 4, then you will find that the value range of $\varphi$ is different, one leads symmetric double rotating arm pattern and the other is single rotating arm pattern.

In order to expressly indicate the above-mentioned theory, insert data of M51 galaxy, and draw corresponding contour line in $Z=0$ plane, the mechanism of symmetric double rotating arm pattern will be clear at a glance. The patterns of its rotating arm in 3D space and $Z=0$ plane are as shown in Figure 15 and Figure 16.

Here it is, the reason that why most spiral galaxies present symmetric double rotating arm pattern. Actually, the law followed by the spiral galaxy is the most spontaneous and simplest natural law.

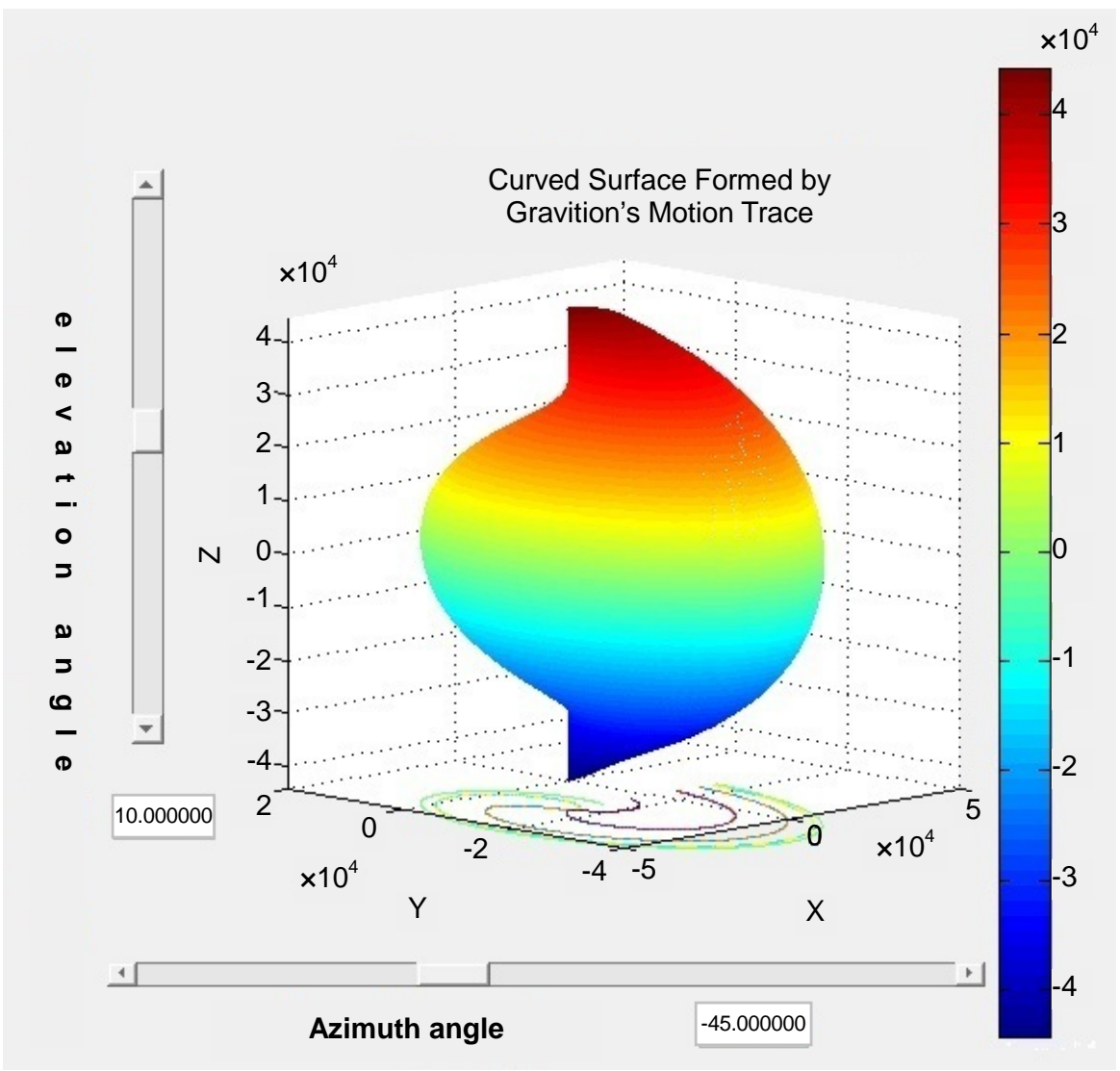

Figure 13. Curved surface formed by motion trace of graviton of spiral galaxy in 3D space when $\varphi \in[0, \pi]$. Unit: light year. 


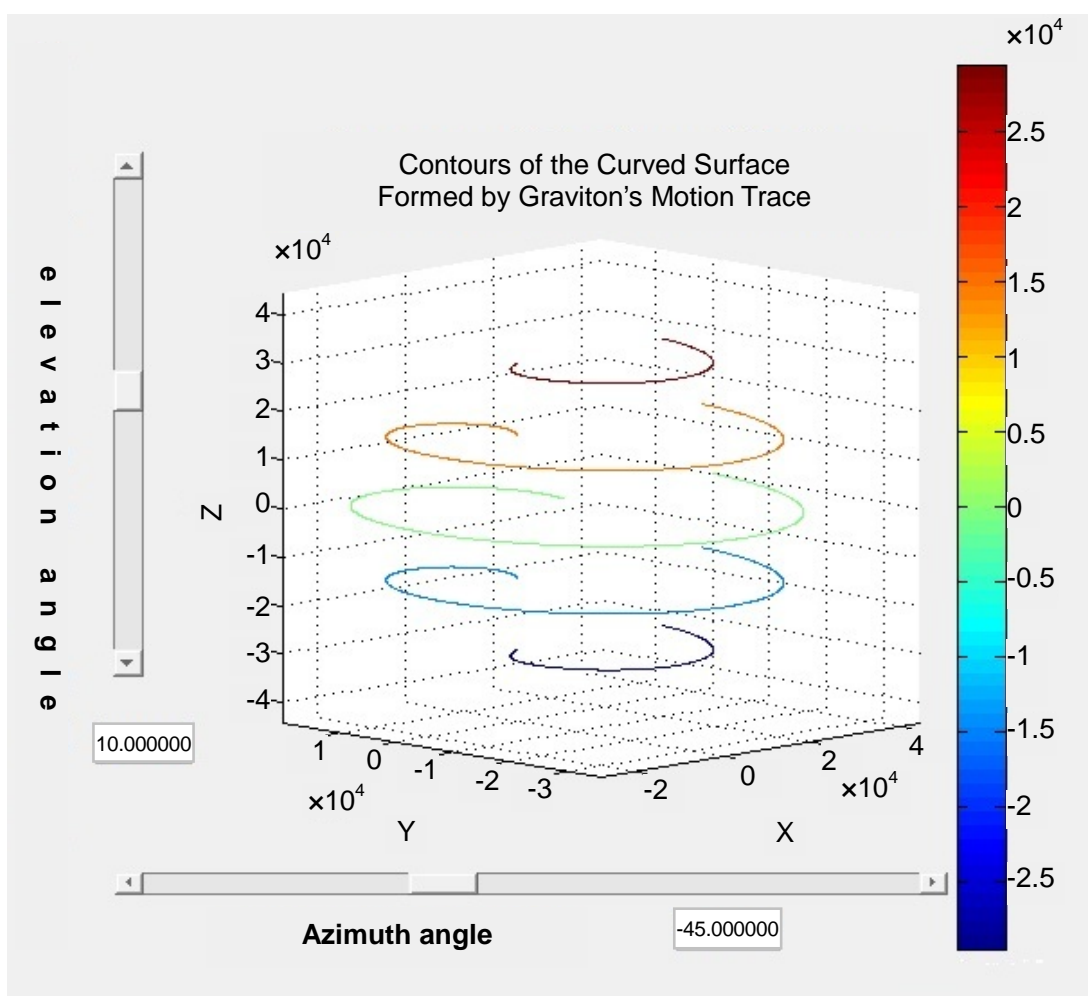

Figure 14. Contour line in curved surface formed by motion trace of graviton of spiral galaxy in 3D space when $\varphi \in[0, \pi]$. Unit: light year.

(a) In 3D, Curved Surface Formed by Gravition's Motion Trace

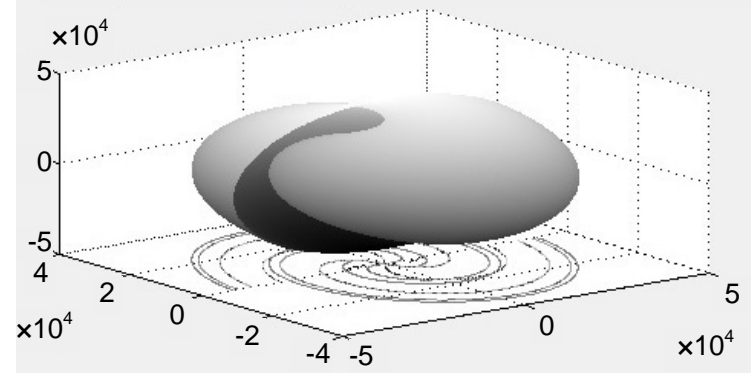

$\times 10^{4}$ (b) When $Z=0$, Contours of the Curved Surface

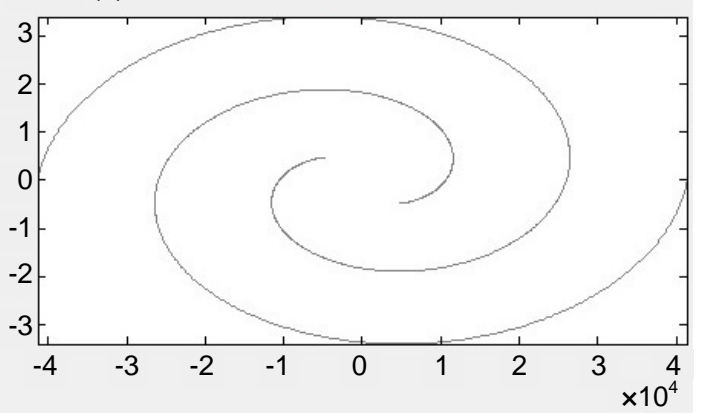

Figure 15. Motion trace of graviton of M51 spiral galaxy and contour line in $Z=0$ plane in 3D space when $\varphi \in[0,2 \pi]$.
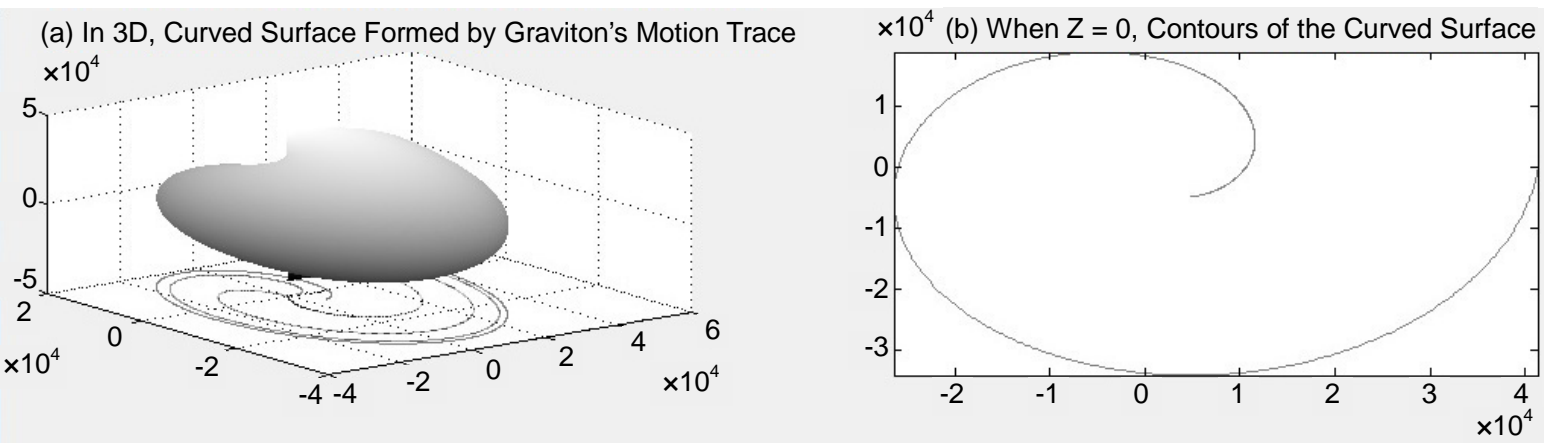

Figure 16. Motion trace of graviton of M51 spiral galaxy and contour line in $Z=0$ plane in 3D space when $\varphi \in[0, \pi]$. 


\section{Graviton Motion Trace and Spiral Galaxy Thickness Distribution after Considering Condition for Load-Carrying Equilibrium in Dynamics of Celestial Body in the Three-Dimensional Space}

In the previous text discusses motion trace of graviton of spiral galaxy. Viewed from ideal condition, stars rotating around the equivalent center of rotation galaxy are ought to distribute nearby motion trace of graviton. But because there is universal gravitation between the equivalent center of rotation galaxy and stars, it needs satisfy condition for force equilibrium in dynamics. Therefore, not all graviton motion trace can stabilize and remain many stars nearby them. For purpose of probing into the influence of kinetic equilibrium, we set up the coordinate system as shown in Figure 17.

Supposed that, the quality of the equivalent center of rotation galaxy is $\mathbf{M}$, and it is located in the original point $\mathrm{O}$ of coordinate system; the quality of stars rotating around the equivalent center of rotation galaxy is $\mathrm{m}$, and it is located in the point $\left(V_{\text {graviton }} \Delta t, \varphi, \theta\right)$ of coordinate system; the angular rotation velocity of the equivalent center of rotation galaxy is $\omega$; the angular rotation velocity that $m$ rotates around the equivalent center of rotation galaxy is $\omega_{m} ; \boldsymbol{r}$ is vector between the original point $\mathrm{O}$ and the location of $m$, and $r_{x y}$ is the projection of $r$ in XOY plane; $h$ is $t$ projection of $r$ in $Z$ axis of the coordinate axis, and the displacement vector to XOY plane.

$$
\left\{\begin{array}{l}
F_{\text {gravitation }}=G \frac{m M}{r^{2}} \\
F_{\text {centripetal force }}=m \omega_{m}^{2} r_{x y} \\
F_{\perp}=F_{\text {gravitation }} \cdot \frac{h}{r}
\end{array}\right.
$$

Here, $G$ is gravitation constant, $r=|\boldsymbol{r}|=V_{\text {graviton }} \cdot \Delta t$.

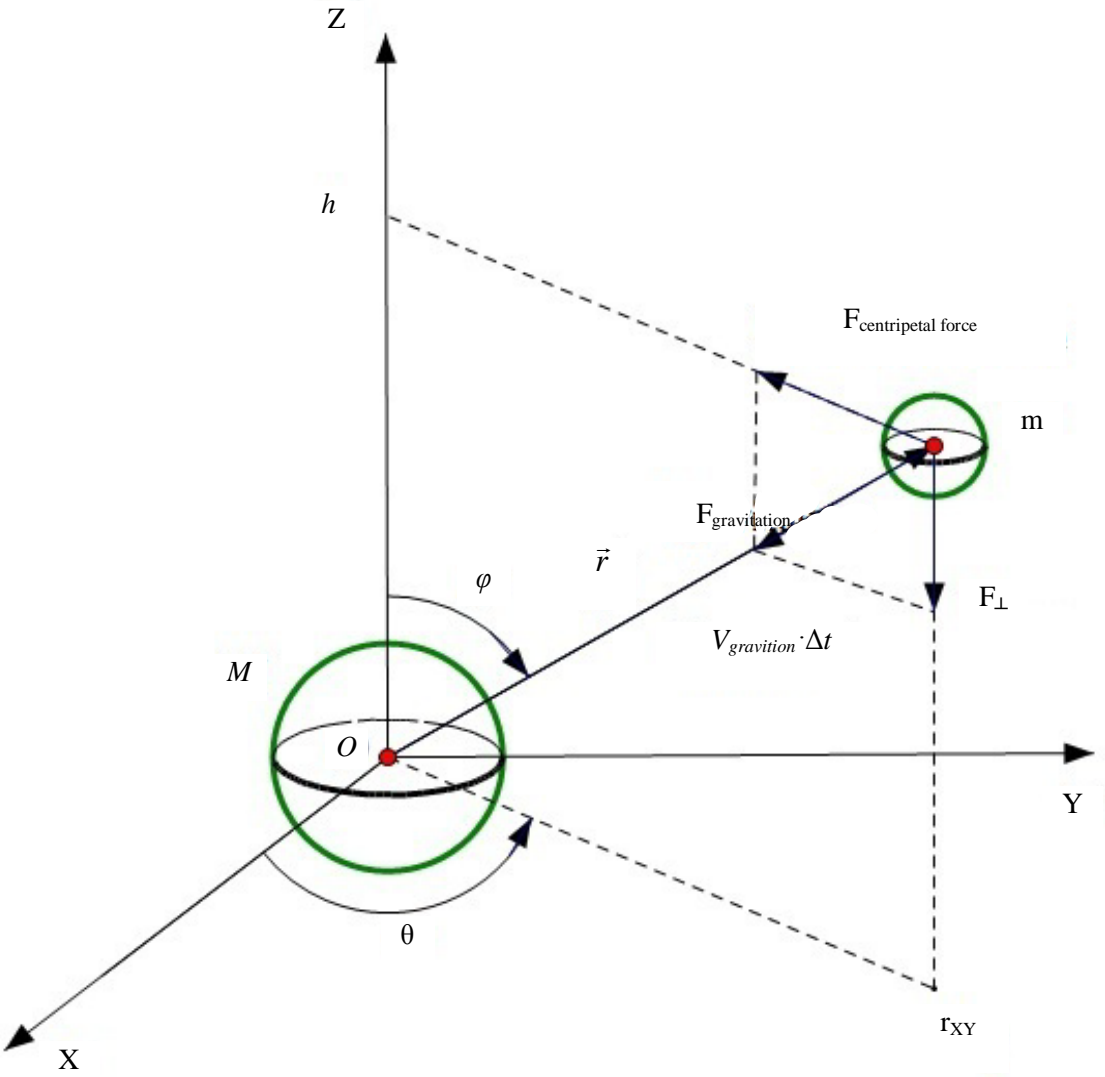

Figure 17. Force analysis of equivalent center of spiral galaxy and star $\mathrm{m}$ in 3D space. 


\subsection{Condition of Stable Equilibrium for Paralleling to XOY Plane Direction}

For paralleling to XOY plane direction, its condition of stable equilibrium is: The formula (14) is equal to the formula (15).

$$
\begin{aligned}
& m \omega_{m}^{2} r_{x y}=G \frac{m M}{r^{2}}|\sin \varphi| \\
& \omega_{m}^{2} r_{x y}=G \frac{M}{r^{2}}|\sin \varphi|
\end{aligned}
$$

In the two-dimensional space, when $\varphi=90^{\circ}, r_{x y}=r$ the formula (17) is simplified into the following formula as,

$$
\omega_{m}^{2} r_{x y}^{3}=G M
$$

The formula (18) is the famous Kepler's Third Law, i.e., in the two-dimensional space, the manifestation pattern of stable equilibrium condition (17) is just Kepler's Third Law. That is to say, astronomical phenomena which can be explained by stable equilibrium condition (17) and traditional Kepler's laws will not cause any conflict; as for generated new laws in the three-dimensional space, please see the following in-depth research.

\subsection{Relation between Thickness $h$ of Galaxy Astrolabe and Distance $r_{x y}$ between It and Galactic Center}

Acquire by the formula (17),

$$
\begin{gathered}
r_{x y}=G \frac{M}{\omega_{m}^{2} r^{2}}|\sin \varphi|=G \frac{M}{\omega_{m}^{2}\left(\frac{h}{\cos \varphi}\right)^{2}}|\sin \varphi| \\
h= \pm \sqrt{G M \frac{1}{r_{x y}} \cdot \frac{\cos ^{2} \varphi \cdot|\sin \varphi|}{\omega_{m}^{2}}} \\
h= \pm \sqrt{G M} \cdot \sqrt{\frac{1}{r_{x y}}} \cdot \frac{\left(\cos ^{2} \varphi \cdot|\sin \varphi|\right)^{\frac{1}{2}}}{\omega_{m}} \\
\because|\cos \varphi| \leq 1, \quad|\sin \varphi| \leq 1 \\
\therefore h \leq \sqrt{G M} \cdot \frac{1}{\sqrt{r_{x y}} \cdot \frac{1}{\omega_{m}}}
\end{gathered}
$$

Discussion: 1) The thickness $\max (h)$ of galaxy astrolabe is in direct proportion to the square root of the quality $M$ of galactic center.

2) The thickness $\max (h)$ of astrolabe in the position of $r_{x y}$ is inversely proportional to the square root of distance $r_{x y}$ between it and galactic center.

3) The thickness $\max (h)$ of astrolabe is inversely proportional to angular velocity rotating around the equivalent center of rotation galaxy.

4) Because of $r_{x y} \neq 0$ in the above formula, it requires $\varphi \neq 0$. In programming, it is necessary to note value range of parameters.

Special attention: In the above deduction, the difference between actual rotation trace s of graviton and radius $r$ of its location and $r_{x y}$ is not distinguished especially. In the formula (14), (15) and (16), simply adopt the radius concept of location to replace the concept of actual motion trace. In these two concepts, which one will be adopted to deduce and more accurate in meaning? We would better use experimental data to conduct comparison and correction. Adopting the meaning at present mainly because of considering that, the surface density 
formed by quantity of graviton in unit solid angle conforms to inverse-square rate, and deduction and application is relatively simple. In addition, the angular rotation velocity $\omega_{m}$ in Figure 17 and Figure 18 is that should be the angular rotation velocity of $m$ for rotating around $M$, possibly different from angular rotation velocity $\omega$ of $M$. They have not been distinguished in this section, which may be an error. Supposed that there is a certain relation between these two angular rotation velocities, such as $\omega_{m}\left(r_{x y}, \varphi\right)=f\left(\omega\left(r_{x y}, \varphi\right)\right)$, so maybe in direct proportion, i.e., $\omega_{m}\left(r_{x y}, \varphi\right)=k_{1} \omega$ and $k_{1} \leq 1$. Because the quantitative relation between them has not been found currently, in order to make convenience for numerical analysis, assume that both of them are equal (that is, take $k_{1}=1$ ), and temporarily use $\omega$ to express, with no distinction.

Supposed

$\omega_{m}\left(r_{x y}, \varphi\right)=k_{1} \omega$ and just temporarily take $k_{1}=1$ and insert into the formula (20), then obtain

$$
h \leq \sqrt{G M} \cdot \frac{1}{\sqrt{r_{x y}}} \cdot \frac{1}{\omega}
$$

Here, $G=6.67 \times 10^{-11} \mathrm{~m}^{3} \cdot \mathrm{kg}^{-1} \cdot \mathrm{s}^{-2}$; as for estimation on $M$, adopt data (please see Table 1 in the document [7]) similar to that of M51 rotation galaxy. May as well assuming that the quality of its equivalent center is $M=1.0 \times 10^{10} \mathrm{~m}_{\text {sun }}$, gathered in the range [7] with radius smaller than $1.1 \mathrm{kpc}$; the quality of a sun is $m_{\text {sun }}=1.989 \times 10^{30} \mathrm{~kg}$.

Convert unit of length in the constant $G$ into light year,

$$
G=6.67 \times 10^{-11} /\left(3 \times 10^{8} \times 365 \times 24 \times 3600\right)^{3}=7.8767 \times 10^{-59}
$$

(light year ${ }^{3} \cdot \mathrm{kg}^{-1} \cdot \mathrm{s}^{-2}$ ). Take angular rotation velocity $\omega_{\text {sun }}$ of the sun as the upper limit value of angular rotation velocity $\omega$ of equivalent center of rotation galaxy, i.e.,

$$
\omega_{\max } \leq \omega_{\text {sun }}=\frac{2 \pi}{25 \times 24 \times 3600} \approx 2.9089 \times 10^{-6}(\text { radian } / \mathrm{s})
$$

Put these data into the formula (21), getting the relation between thickness $h$ of M51 rotation galaxy and $r_{x y}$ as,

$$
\begin{gathered}
h \leq 1.2517 \times 10^{-9} \frac{1}{\sqrt{r_{x y}}} \cdot \frac{1}{\omega} \\
h \leq 4.3029 \times 10^{-4} \cdot \frac{1}{\sqrt{r_{x y}}}
\end{gathered}
$$

Unit: $h$ : light year, $r_{x y}$ : light year, $\omega$ : radian/s.

Attention,

1. When $r_{x y}$ is smaller than some value (supposed that this value is $r_{\min }$ ), there should be another constraint condition.

2. From the formula (22) to (23), adopt $\omega_{\max }$. So, strictly speaking, from mathematical derivation, $\leq$ in the formula (23) will not be established. But in order to embody that physical meanings accommodating stars is allowed in the trace smaller than $h\left(r_{x y}\right)$ trace in mathematical expression, this writing style is remained temporarily, and not traced strictly. After obtaining the minimum value $\omega_{\min }$ of $\omega$ or real $\omega$, insert it into the formula (22) again, the formula (23) obtained from this will be established in really strict meaning. At present, there is no way to gain numerical expression, conducting comparison by images.

3. In programming and calculating, please not that $r_{x y} \neq 0$.

Combining constraint conditions in the formula (20) or (21) with the formula (5), (6) and (7), we gets the image that stars of spiral galaxy are all located in thin disk, which accords with practical situation very well. So as to display this beautiful image, especially inset observation data of M51 galaxy into the above-mentioned formula, then gain a group of formulas which can be shown in figure, such as the formula (10), (11), (12) and (23) discussed previously. Its 3D image can be drawn by using Matlab software, as shown in Figure 18 and Figure 19. 


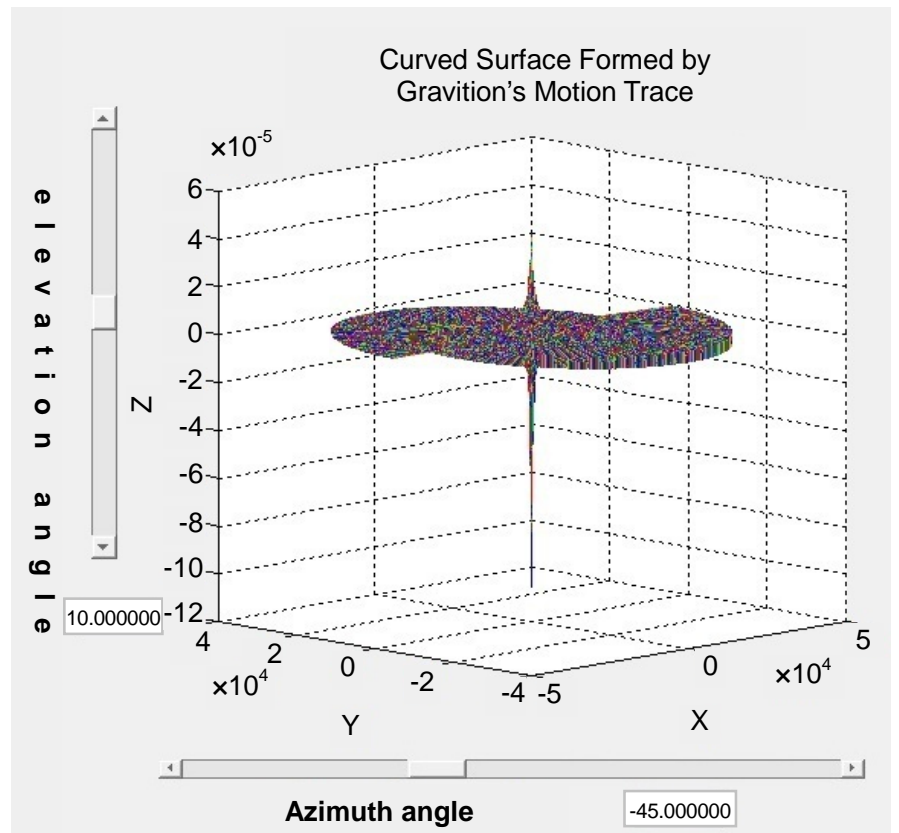

Figure 18. 3D motion trace of graviton of spiral galaxy in the condition of physical constraint. Unit: light year.

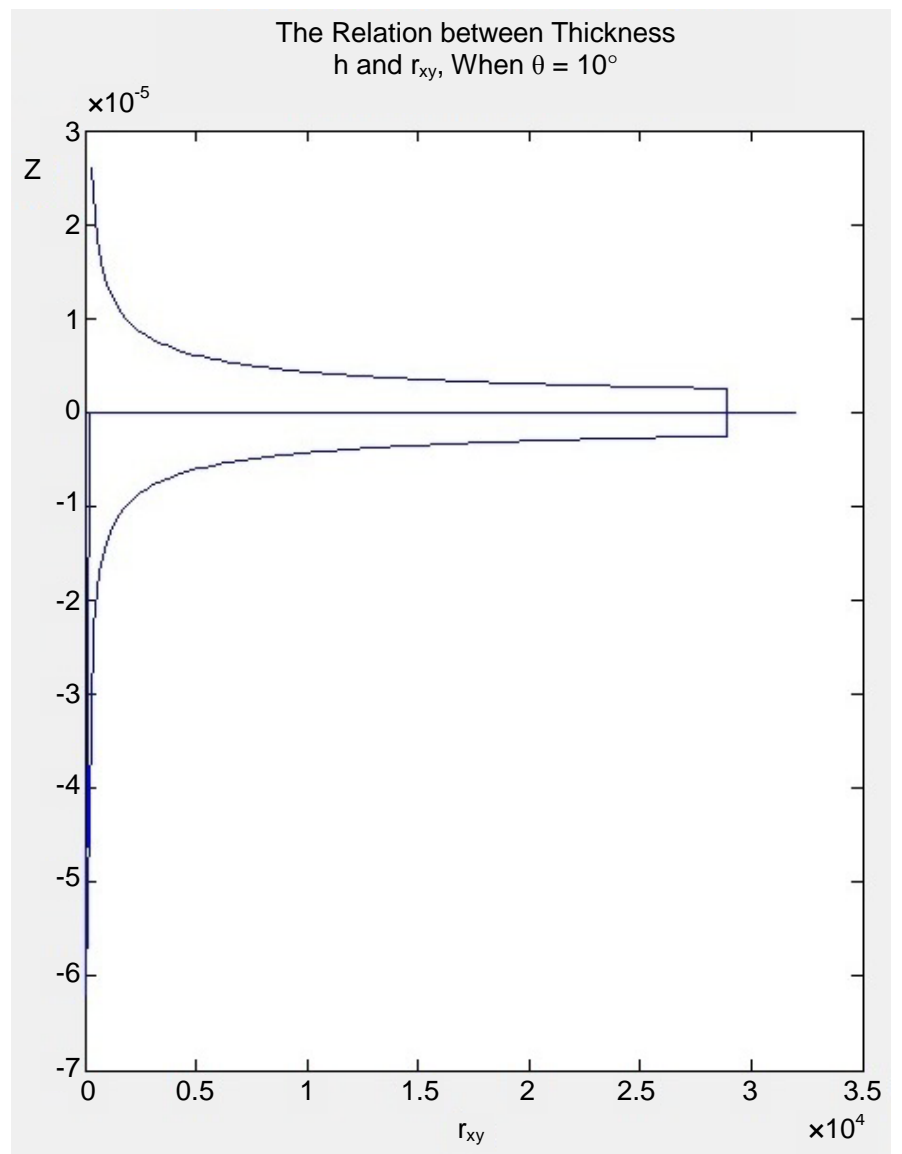

Figure 19. Profile diagram of 3D motion trace of graviton of spiral galaxy in the condition of physical constraint. Unit: light year. 


\subsection{The Comparison with the Observation Image and Current Mainstream Theoretical Model Law}

Applying the above-mentioned theory that the thickness of astrolabe is inversely proportional to the square root of distance $r_{x y}$ between it and galactic center into image of NGC891 galaxy (see Figure 20), we can find that the theoretical fitting curve accords with actual fitting curve very well. It shows that this theory is right, as a great success.

In figure, the horizontal and vertical red line, representing two symmetric axises that are meristic with the center of NGC891, which are used to establish coordinate system. The vertical line is $Z$ axis, the horizontal line is $r_{x y}$ axis and the cross point is the original point. The white curved line is fitted by the formula (20).

There is a question, that is, the present popular theory also put forwards a exponent distribution model [12], so which one is better?

The fitting formula of exponent distribution model raised by the present popular theory can be denoted by the formula (24).

$$
h=k_{2} \cdot \mathrm{e}^{-c_{2} r_{x y}}
$$

We conduct the same data processing in allusion to data in Figure 20, and draw them in the same image to carry out comparison. The result is as shown in Figure 21.

Viewed from Figure 21, the fitting curve of graviton motion trace theory better accords with the external profile of galaxy and hand-painted curve. From model simplification and physical meanings of parameters, the physical thought of graviton motion trace theory is comparatively simple, the formula can be directed made from established coordinate system and the physical meanings of parameters are clear, which meet "the principle of simplification”. Apply thought that field quantum transmits acting force, put forward graviton motion trace and use basic physics theory to add dynamic constraint on the theory of graviton motion trace, overcoming "incompatibility of microphysics and macroscopic physics", realizing integration of knowledges in relevant fields and satisfy "the principle of harmony". In the condition of using several galaxy images with great different in shape to conduct verification, the coincidence degree of the theory and actual measurement image is high, satisfying "the principle of testability". Other testing principles all can be verified gradually in the following researches in success. According to "The criterion of both high and low quality among a group of theories" [13], the theory of graviton motion trace owns greater superiority.

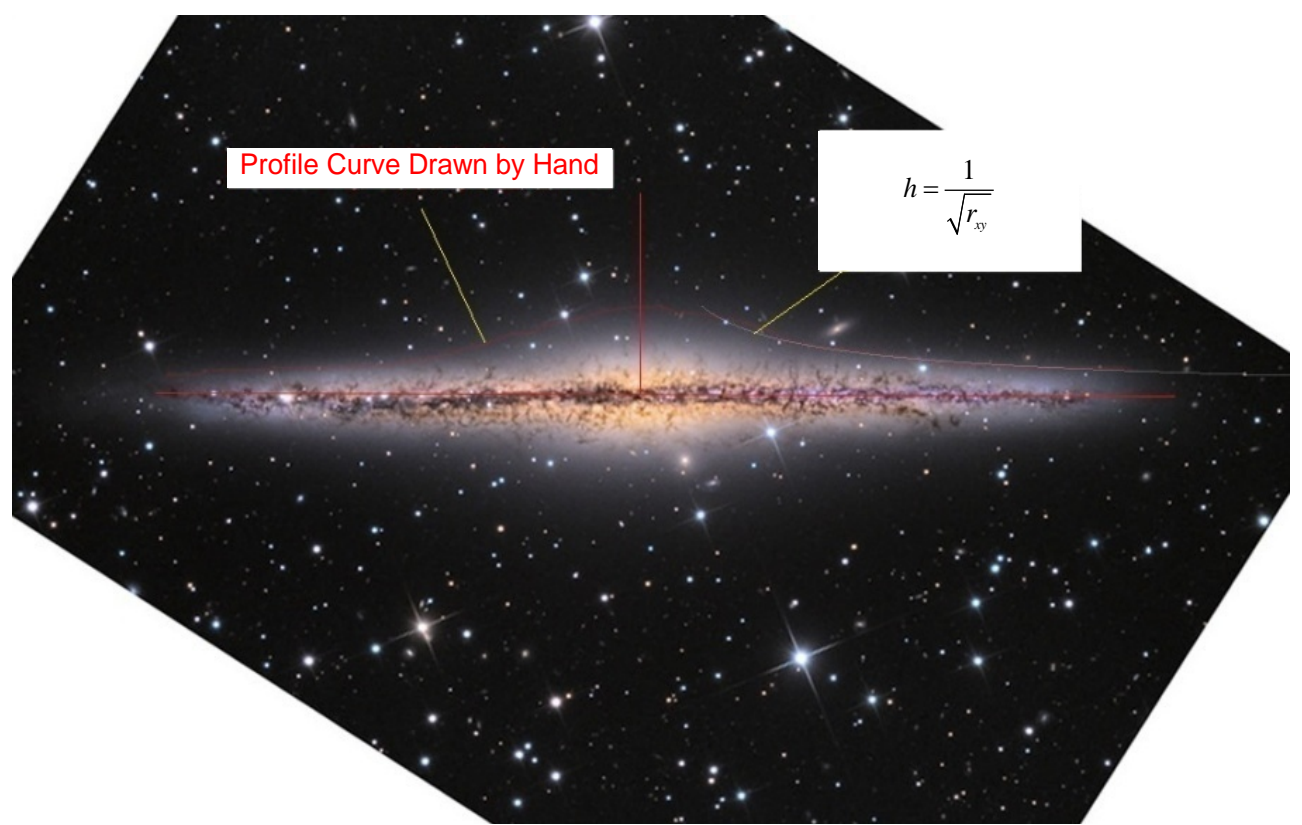

Figure 20. The thickness of astrolabe is inversely proportional to the square root of distance $r_{x y}$ between it and galactic center in NGC891 galaxy. 


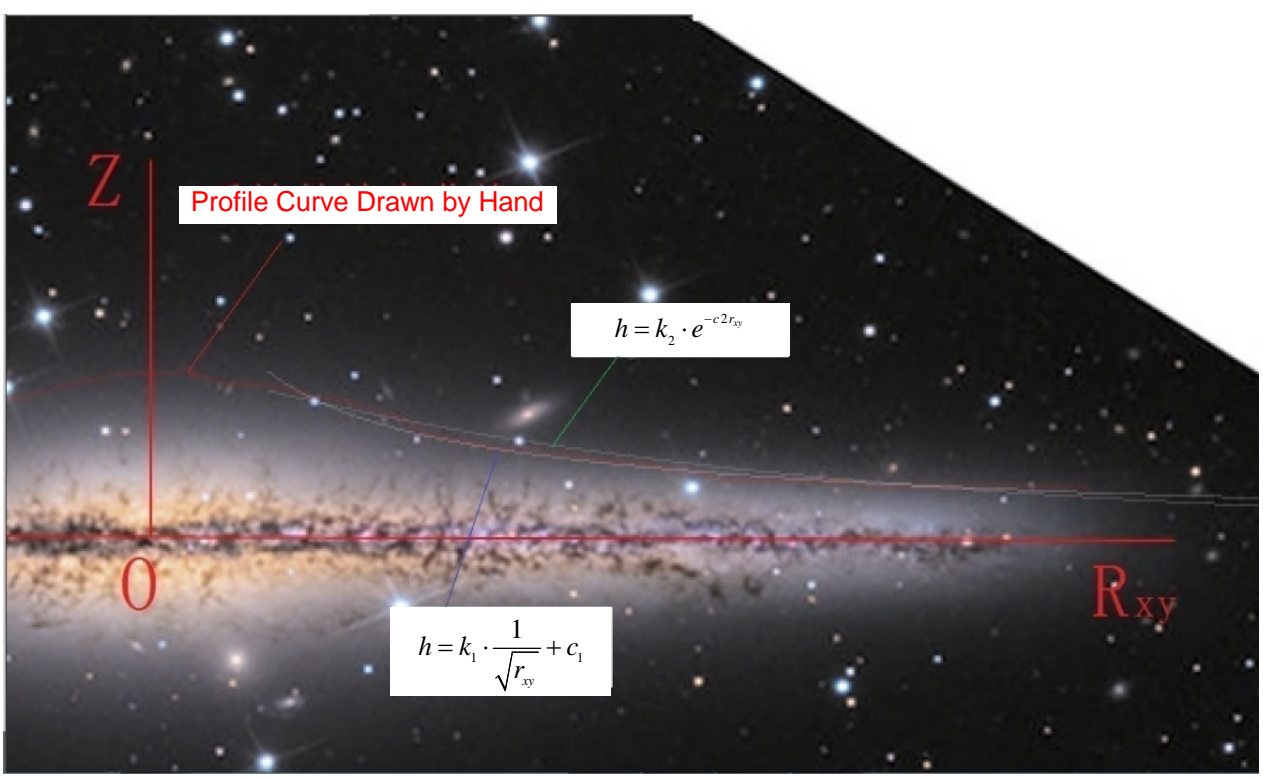

Figure 21. Comparison between two model fitting curves and hand-painted curves (outline of galaxy) in NGC89 galaxy.

In addition, the document [14] reports a piece of scientific news, "Some spiral galaxies are plump and ridgy, while some others like flat disk. Galaxies seem so different with each other, which has been always a puzzle filled with controversy.” Recently, an international astronomer group from Australia has discovered that, it depends on their rotation velocity, that is, the spiral galaxy rotating fast is flat and thin, while the galaxy with the same size rotating slowly is fat and plump. As per the formula (22), $h \propto \frac{1}{\omega}$, i.e., there is the law of "that the galaxy thickness $h$ is inversely proportional to angular rotation velocity $\omega$ ", which accords with actual phenomenon reported above. Due to that the original research paper has not been found, specific comparison has not been made here temporarily.

\section{Conclusions}

1) On the basis of motion law of graviton of spiral galaxy in the two-dimensional space proposed in the document [4] [5], this article raises motion law model of graviton of spiral galaxy in the three-dimensional space, revealing internal essence of formation of spiral galaxy. It tactically combines microscopic graviton motion law and macroscopic galaxy pattern, overcoming lots of disadvantages of the current popular theories.

2) The combination, between motion law model of graviton of spiral galaxy in the three-dimensional space and dynamic constraint condition, is able to naturally reveal mechanisms of symmetric double rotating arm structure and thin disk structure of spiral galaxy.

3) Through applying theories in this article, analyzing image of galaxy greatly differing from M51, such as M101 and Hoag's Object shown in Figure 12, as per images in polar coordinate system which these images correspond to, it is verified again that, the shape of rotating arm presents law $r \propto \theta$, i.e., the rotating arm of spiral galaxy conforms to Archimedes spiral in the shape (it is not given out due to limitation of article length. And independent verification on it is welcomed), but not the present mainstream viewpoint (the shape of rotating arm accords with the logarithmic spiral linetype [15]), which is not an accidental result. Therefore, it further verifies the correctness of this theory.

4) The changes of different parameters in the theory of this article can result in galaxy images with great difference in shape, and the actual picture of corresponding galaxy image can be found in observation image, which indicate that this theory owns universality.

5) The law that galaxy thickness changes with radius deduced theoretically in this article, is not only simple in model and clear in physical meaning, but also that its precision is better than exponential decay law [12] proposed by the present mainstream theory in accordance with verification of NGC891 galaxy observation picture. 


\section{Acknowledgements}

Special thanks to Professor Ye Zhiyao of Beijing Chemical Defense Institute for encouragement and support, also Professor Cao Shenglin, a astronomy expert from Beijing Normal University, Chen Yue, Wu Yuejiang, Zhu Hongbo, Meng Xiaodong and Zhao Caifeng, former graduate students from Department of Physics, Beijing Normal University, putting forward lots of valuable advices in the research process of this project.

\section{References}

[1] Adam, T., Agafonova, N., Aleksandrov, A., Altinok, O., Alvarez Sanchez, P., Anokhina, A., et al. (2011) Measurement of the Neutrino Velocity with the OPERA Detector in the CNGS Beam. http://arxiv.org/abs/1109.4897

[2] Brumfiel, G. (2011) Particles Break Light-Speed Limit. http://www.nature.com/news/2011/110922/full/news.2011.554.html

[3] The Scientists Affirm That Speed of Neutrino Isn’t Super Velocity of Light and Still Submits to the Einstein's Theory 2012. http://tech.sina.com.cn/d/2012-06-13/09067262117.shtml

[4] Luo, P. (2012) Journal of Modern Physics, 3, 297-303.

[5] Luo, P. (2013) Journal of Modern Physics, 4, Article ID: 41152.

[6] Min, W. and Xu, K. (2007) Progress in Astronomy, 25, 215-225.

[7] Chen, Z. and Williams, P. (2006) Progress in Astronomy, 24, 319-334.

[8] Shen, Y.H., Liang, Z.Z., et al. (2001) Practical Mathematical Manual. Science Press, Beijing, 79.

[9] Pixastro’s Website, Barred Spiral Galaxy NGC1300. http://zhan.renren.com/pixastro

[10] Baidupedia, Hoag's Object. http://baike.baidu.com

[11] Yuan, Y.Z. (1984) Progress in Astronomy, 2, 230-237.

[12] Peng, Q.-H., Luo, X.L., et al. (2001) Progress in Astronomy, 19, 397-411.

[13] Luo, P. (2011) The Criterion of both High and Low Quality among a Group of Theories. http://blog.sina.com.cn/s/blog 4bd628330100uze0.html

[14] Ling, H. (2014) The Ridge or Flat in Galaxies Depends on Their Rotation Velocity. Science and Technology Daily, March 17, 2014.

[15] Luo, X.L., Peng, Q.-H., et al. (2000) Acta Astronomica Sinica, 41, 424-432. 
Scientific Research Publishing (SCIRP) is one of the largest Open Access journal publishers. It is currently publishing more than 200 open access, online, peer-reviewed journals covering a wide range of academic disciplines. SCIRP serves the worldwide academic communities and contributes to the progress and application of science with its publication.

Other selected journals from SCIRP are listed as below. Submit your manuscript to us via either submit@scirp.org or Online Submission Portal.
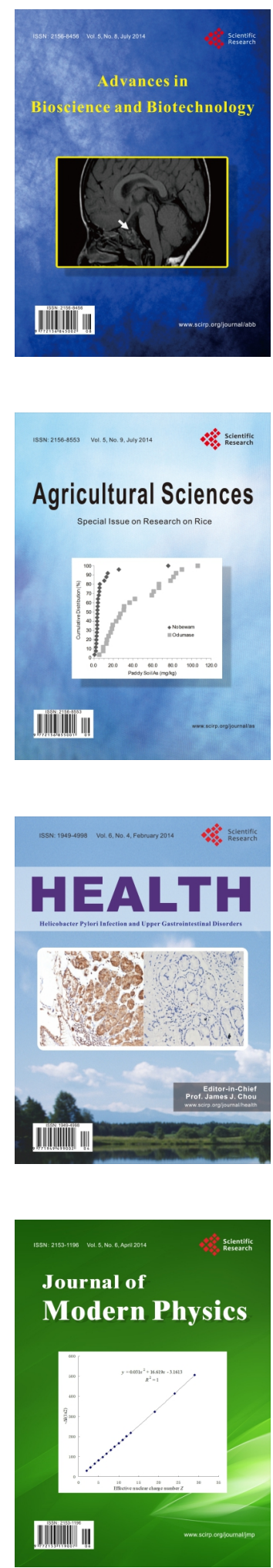
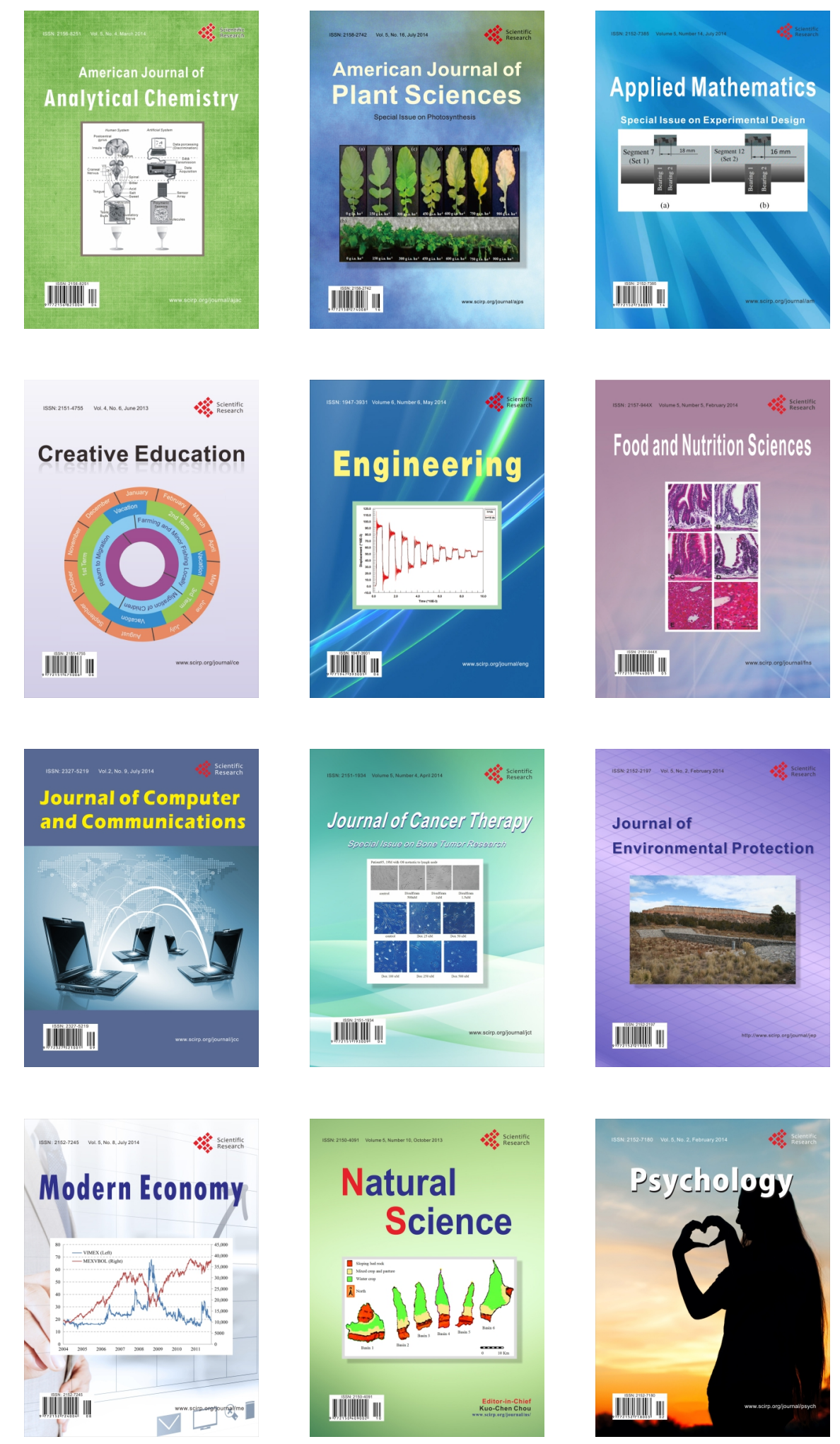